\title{
1 The annotation and analysis of complex 3D plant organs using
}

\section{3DCoordX}

3

4 Athul Vijayan ${ }^{1, \# a, \text {, Soeren Strauss }}{ }^{2, \pi}$, Rachele Tofanelli ${ }^{1}$, Tejasvinee Atul Mody ${ }^{1}$, Karen

5 Lee $^{3}$, Miltos Tsiantis ${ }^{2}$, Richard S. Smith ${ }^{2,3}$ and Kay Schneitz,*

6 Plant Developmental Biology, TUM School of Life Sciences, Technical University of

7 Munich, Freising, Germany

8 2Department of Comparative Developmental and Genetics, Max Planck Institute for Plant

9 Breeding Research, Cologne, Germany

${ }^{3}$ The John Innes Centre, Norwich Research Park, Norwich, United Kingdom

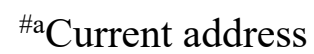

12 Department of Comparative Developmental and Genetics, Max Planck Institute for Plant

13 Breeding Research, Cologne, Germany

*Corresponding author

Email: kay.schneitz@tum.de (KS)

TThese authors contributed equally to this work.

Short title: Computational cell annotation in 3D digital plant organs

Email addresses authors:

Athul Vijayan: avijayan@mpipz.mpg.de

Soeren Strauss: strauss@mpipz.mpg.de

Rachele Tofanelli: r.tofanelli@tum.de

Tejasvinee Atul Mody: t.mody@tum.de

27 Karen Lee: karen.lee@jic.ac.uk

Miltos Tsiantis: tsiantis@mpipz.mpg.de

Richard Smith: Richard.Smith@jic.ac.uk 


\section{Abstract}

35 A fundamental question in biology concerns how molecular and cellular processes become integrated during morphogenesis. In plants, characterization of 3D digital representations of organs at single-cell resolution represents a promising approach to addressing this problem. A major challenge is to provide organ-centric spatial context to cells of an organ. We developed several general rules for the annotation of cell position and embodied them in 3DCoordX, a user-interactive computer toolbox implemented in the open-source software MorphoGraphX. It enables rapid spatial annotation of cells even in highly curved biological shapes. With the help of 3DCoordX we obtained new insight by analyzing cellular growth patterns in organs of several species. For example, the data indicated the presence of a basal cell proliferation zone in the ovule primordium of Arabidopsis thaliana. Proof-of-concept analyses suggested a preferential increase in cell length associated with neck elongation in the archegonium of Marchantia polymorpha and variations in cell volume linked to central morphogenetic features of a trap of the carnivorous plant Utricularia gibba. Our work demonstrates the broad applicability of the developed strategies as they provide organ-centric spatial context to cellular features in plant organs of diverse shape complexity.

\section{Introduction}

53 It remains a salient challenge to understand the generation of biological shape. Gaining comprehensive insight into the multi-scale processes underlying morphogenesis critically depends on the quantitative description of molecular, cellular, and tissue-level parameters, such as gene and protein expression, cell geometry, and cell topology 

can be achieved by growth that is oriented relative to a coordinate system imposed on the organ [7]. Thus, insight into tissue morphogenesis further relies on putting cellular data into context by placing them within an organ-related frame of reference [8-11].

61

62 Realistic 3D digital organs with cellular resolution have become indispensable tools for the study of morphogenesis. They can be obtained by deep imaging of fluorescently marked specimens using for example confocal laser scanning microscopy (CLSM) or light sheet fluorescence microscopy (LSFM) followed by 3D cell segmentation of the obtained z-stacks of optical sections with the help of constantly improving software [1117]. Tissues and organs of model plants are particularly well suited for the generation of such 3D digital representations. Plant cells are immobile simplifying the detection of cellular growth patterns associated with tissue formation. In addition, plant tissues are characterized by a small number of different cell types and often exhibit a well-structured, layered organization. Thus, they usually feature a cellular anatomy of manageable complexity. Accordingly, a growing number of realistic 3D digital tissues with cellular resolution are being generated, mainly in the model plant Arabidopsis thaliana $[9,10,18-$ 27].

With the help of 3D digital organs quantitative information about geometric and molecular parameters of up to thousands of cells can readily be obtained using opensource software, such as MorphoGraphX [11,14] (preprint). However, meaningful exploration of such complex data sets remains challenging. In particular, it is important to provide spatial context by placing the cell's data within an organ-related frame of reference [8]. Several computational pipelines have been established that provide such a 
tissue-level frame of reference and allow the semi-automatic annotation of 3D cellular properties in a plant tissue context with cellular resolution: iRoCS [9], 3DCellAtlas [10], and 3DCellAtlas Meristem [22]. These computational pipelines have been applied very successfully for the annotation of cells and tissues in the main root and the hypocotyl, radially symmetric organs with limited curvature, or the SAM, a dome-shaped structure exhibiting an anatomy of moderate complexity. However, not all plant organs fall into these simple morphogenetic categories. For example, strong curvature caused by developmentally regulated differential growth limits the usefulness of the implemented analytical strategies in iRoCS and 3DCellAtlas, particularly for indexing the axial position of a cell and determining its absolute distance to a reference. Yet, curvature represents a central element of the morphogenesis of plant organs with complex 3D shapes [28]. Many plant organs exhibit varying degrees of curvature, for example the apical hook of seedlings, leaves, or floral organs, such as sepals or petals. The ovule, the major female reproductive organ in higher plants, constitutes a particularly prominent example. Ovules are characterized by complex anatomy consisting of a central "trunk", made up of several functionally distinct tissues stacked on top of each other, and by one or two laterally attached integuments, determinate planar tissues that eventually develop into the seed coat [29]. Moreover, most angiosperm ovules exhibit an extreme curvature due to asymmetric growth of the integuments [30].

Here, we used the ovule of Arabidopsis thaliana as a model to develop new generic strategies, implemented in MorphoGraphX, that allow the straightforward establishment of intrinsic coordinate systems in organs of simple or elaborate shapes. To this end we took advantage of a recently generated digital 3D reference atlas of ovule development in Arabidopsis [27]. We illustrate how such a coordinate system enables rapid annotation of 
cell identity and cell position in 3D and greatly facilitates the quantitative analysis of

Finally, we demonstrate the broad applicability of the introduced concepts by providing

111 proof-of-concept analysis for selected parameters in different plant organs of varying

112 shape complexity.

\section{Results}

\section{Curvature-related complications in the assignment of axial}

\section{6 position}

117 The analytical strategies for the positional annotation of individual cells relative to tissue organization depend on the structure under study. For example, the straightforward approaches employed in iRoCS and 3DCellAtlas involve cylinder coordinates and work very well for indexing axial position of cells relative to a reference in the root or hypocotyl, structures exhibiting limited curvature (Fig. 1A). However, asymmetric growth caused by differential cell proliferation and/or cell expansion can result in slanted or highly curved organs. In these instances, such approaches may lead to cells of the same indexed position having different absolute axial distances to a common reference (Fig. 1B). Thus, we devised a different strategy to minimize such axial distance errors when assigning 3D positional annotation to cells of organs that exhibit complex shapes. Our approach takes cues from central patterning events that frequently occur during early plant organogenesis, in particular the distinction of radial layers, the subdivision into anterior-posterior domains, and the establishment of a proximal-distal (axial) distance field (Fig. 1C,D). The proximal-distal distance of a cell relative to a user-defined 
reference is estimated by finding the shortest path through the cell centroids. Importantly, the search is confined to a given tissue layer and may not cross the anterior-posterior domain. The restriction to a tissue layer removes a large part of the axial distance error as

134 the shortest path through the tissue layers cannot extend through interior tissues (Fig. 1C).

135 On top of this restriction, prohibiting the shortest path from passing the anterior-posterior boundary further minimizes the error (Fig. 1D).

\section{Development of the Arabidopsis ovule}

139 The typical angiosperm ovule represents a prime example of an elaborately curved structure. Ovule development in Arabidopsis thaliana is well described [24,27,31,32]

141 (Fig. 2A,B). During stage 1 the ovule emerges as a finger-like protrusion from the placenta (staging according to [27,32]). Eventually, three elements can eventually be recognized along the trunk of the developing ovule: the nucellus at its tip, the chalaza in the center, and the funiculus at the bottom. The nucellus generates the large subepidermal megaspore mother cell (MMC) that will undergo meiosis during stage 2. sac or female gametophyte carrying the egg cell proper. The chalaza is characterized by two epidermally-derived integuments, lateral tissues that initiate from its flanks during stage 2 . The outer integument represents a bilayered structure while the inner integument eventually consists of three cell layers. The two integuments grow around the nucellus but leave open a small cleft, the micropyle, through which the pollen tube can reach the embryo sac. The funiculus, a stalk-like structure, harbors the vascular strand and connects the ovule to the placenta. The mature Arabidopsis ovule features an elaborately curved shape. Curvature is caused in part by the integuments bending around the nucellus during 
funiculus forms a bend as well. Overall, the mature ovule exhibits a characteristic doublycurved structure (Fig. 2A).

\section{Developmental axes of the ovule primordium}

The Arabidopsis ovule primordium exhibits the typical radial organization into the L1, developing ovule. Gene expression patterns underlying the proximal-distal organization of the primordium are relatively well understood $[27,35,36]$. Importantly, the early primordium is not growing in a straight fashion but rapidly adopts a slant relative to the placenta surface, with the small angle of the slant facing the septum. It represents the first morphological sign of an anterior-posterior polarity (Fig. 1C,D) [27]. The presence of an anterior-posterior axis is further supported by the anterior expression of the class III HDZIP gene PHABULOSA in the early primordium [37]. To corroborate the establishment of an anterior-posterior axis in the stage 1-I ovule primordium we investigated the spatial signal distribution of pKAN1::KAN1:2xGFP, a reporter for KANADI1 expression [38]. primordium (Fig. 2C). Interestingly, we did not detect expression in the tip of the primordium. By stage 2-I reporter signal appeared to be restricted to the posterior epidermis of the prospective funiculus.

In summary, the combined evidence strongly suggests the establishment of a radial, a

178 proximal-distal, and an anterior-posterior axis in the ovule primordium. At the same time, 179 the slant represents an early morphological manifestation of the ovule becoming a curved structure. 


\section{Cell layer detection, individual organ separation, and anterior-}

\section{posterior domain annotation in $3 D$}

The annotation of cell position in $3 \mathrm{D}$ in the slanted ovule primordium required the

application of the general principles outlined above. To be able to do so in a fast and

robust manner we devised a new method for radial tissue labeling. Current pipelines, such

as 3DCellAtlas and 3DCellAtlas Meristem, invoke a surface mesh as a central tool for

cell and tissue annotation in the root, hypocotyl, and the SAM [10,22]. However, placenta (Fig. 3A). The resulting individual surface meshes fail to outline the surfaces of the cells in contact and it is labour intensive to recreate a surface mesh in such instances.

To address this problem, we developed a new strategy to perform automatic layer detection that groups cells into L1, L2, and L3 without a need for a surface mesh (Fig. 3A-C). In a first step L1 cells are clustered on the basis of a cell at the outer surface of the organ not being flanked by a neighboring cell at their outer surface. This feature is captured by defining the ratio of unshared wall area to shared wall area of individual cells (outside wall area ratio). Once L1 cells are clustered, L2 and L3 cells are found by their relative distances to the $\mathrm{L} 1$ cells. To this end a network of cell centroids is established and the shortest number of centroids a cell must cross to reach to the nearest L1 cell is determined. The corresponding result essentially reveals how many cells separate the cell of interest from the L1. The information can be directly used to cluster cells into L2 and 
neighboring organs are in full contact. However, the process works well when there is partial contact that still leaves behind a significant outside unshared cell wall area. This approach successfully annotated the radial tissue layers in ovule primordium or the shoot apical meristem (Fig. 3A-C, Fig S1).

Another problem relates to the separation of the multiple ovule primordia attached to the placenta into distinct units to allow ovule-specific analyses. We devised a method that takes advantage of the cell connectivity graph (Fig. 3D). From a selected cell at the distal end on each different ovule, distances to all other cells on the cell connectivity graph are computed. Cells are then assigned a label based on their nearest selected cell on that graph. A further parameter sets a maximum size of the ovules (in number of cells from the selected cells) to separate the ovules from their surrounding tissue. To facilitate downstream analysis, different labeling types, such as the cell layers and the ovule labels, are combined to create a unique label for each layer in every ovule.

In the last step, cells of anterior and posterior domains of about similar dimensions are obtained by manual selection (Fig. 3E). For the funiculus we also devised a semiautomatic method to distinguish the anterior and posterior domains (Fig. S2). In summary, the outlined approaches enable the generation of 3D digital ovule primordia of separate identities and near-perfect radial and anterior-posterior tissue annotation with minimal user input.

\section{Assignment of proximal-distal position to individual cells in 3D}


anterior-posterior domains have already been annotated. The proximal-distal position of each cell is calculated, either in terms of cell index or absolute or relative distance to a distance field and is placed at one end of the tissue. In the case of the cone-shaped ovule primordium a small, near point-size Bezier ring is positioned at the distal tip of the primordium. Positioning the Bezier ring at the distal tip correlates with a biologically relevant maximum of the phytohormone auxin at the tip as inferred from the expression of the auxin response reporter pDR5::GFP [39], the spatial signal of the auxin sensor R2D2 [40,41], and the finding that polar auxin transport mediated by PINFORMED1

241 (PIN1) is required for ovule primordium formation [42,43]. In case of the mature

242 funiculus that is close in shape to a curved cylinder, a larger Bezier ring is placed at its $\mu \mathrm{m})$ to the user-specified Bezier ring (Fig. 1C,D). They act as seeds for the distance coordinates of the remaining cells of the tissue that are obtained by searching for the shortest path through the cell centroids to the centroid of an origin cell. The search is restricted to a radial layer and by the anterior-posterior boundary. It should be noted that with this approach small axial distance errors still occur within the anterior or posterior domains depending on the number of laterally arranged cell files within these areas. The remaining errors are typically in the range of a few microns, but can be eliminated when taking individual cell files into account. This is possible within the software, however, the procedure involves cumbersome manual annotation of all cell files for each cell layer. 
The two integuments undergo complex morphogenesis with the inner integument a curved hood-like shell (Fig. 2A,B,F). Both integuments are characterized by their own intrinsic developmental axes (Fig. 2E-H). A distinct adaxial-abaxial (dorso-ventral) axis is prominent as the individual cell layers differ in cellular morphology and gene expression patterns $[32,37,44-46]$ (Fig. 2E). Both integuments also feature their own proximal-distal axes (Fig. 2A,F). Related to its hood-like shape the outer integument flanks a frontal section and features a medial-lateral axis (Fig. 2G). As a rule, we position the ovule with the anterior domain and the micropyle pointing to the left and the proximal end of the funiculus pointing towards the bottom right. Based on this arrangement we define the left and right lateral sides of the medial-lateral axis (Fig. 2H).

We successfully applied to the integuments some of the same formal strategies as described for the primordium or funiculus. In an initial step, the integumentary adaxialabaxial cell layers are labelled manually. In a subsequent step, medial-lateral coordinates of all cells of a given integument layer are established relative to a file of posterior midline cells (Fig. 4A). Cell distance is computed in terms of how many cells separate a given cell from the midline (Fig. 4B). Cells along the medial-lateral axis can be grouped

274 further into median and lateral subdomains that occupy about half the width of an

275 integument layer. For example, for the outer layer of the outer integument we grouped 276 cells that are located three cells to the left or right of the posterior midline cells into the 277 median domain. The remaining cells are classified as lateral cells (Fig. 4C). In the 278 following step, proximal-distal distance coordinates are assigned for all integument cells. 279 A Bezier ring is first placed at the proximal end of the inner side of the outer integument 280 (next to its inner layer) facing the outer layer of the inner integument (Fig. 4D). The 
circular origin is in the same plane as the ring-shaped expression pattern of the

pCUC3::CUC3:CFP reporter which marks the proximal base of the two innermost layers of the inner integument, respectively (Fig. 4E). Members of the CUC gene family are generally required for primordium initiation and organ boundary formation [43,47-52].

Origin cells are then defined by their close user-specified distance to the Bezier ring in 3D (about 5-15 $\mu \mathrm{m}$ ). As a direct result of the placement of the Bezier ring cells of the proximal chalaza are assigned a negative value for the proximal-distal position (Fig. 4F). This feature can be used to separately cluster and analyze those cells. Finally, proximaldistal distance coordinates of the integument cells are obtained by searching for the shortest path through the cell centroids to the centroid of an origin cell (Fig. 4F, G). The search is again restricted to a given tissue layer and may not cross the medial-lateral boundary. Taken together, the procedure assigns medial-lateral and proximal-distal positions for all cells of the integuments.

\section{Differential distribution of cellular growth patterns during}

\section{early ovule development}

To provide proof of concept for the usefulness of our computational tools in the quantitative analysis of cellular patterns in a 3D context we assessed spatial growth patterns in selected aspects of ovule development. To this end we made use of a previously published dataset of wild-type 3D digital ovules of the Col-0 accession [27]. grow in a continuous fashion based on an analysis of the total number of cells and the increase of organ volume from stages 1-I to 2-I [24,27]. However, it remained unclear how cell numbers and cell volumes of the radial layers compare to each other. In addition, 
it was not known if mitoses are randomly distributed along the proximal-distal axis or if

307 they preferentially occur in specific domains. To address these questions we analyzed 52

3D digital wild-type ovule primordia that encompassed stages 1-I to 2-I (Fig. 5A,B).

310 We initially undertook a comparison of cell volumes between stages (Fig.5C) and radial layers (Fig. 5D) (Table 1). In this dataset the L2-derived MMCs at stage2-I feature an average cell volume of $543.3 \mu \mathrm{m}^{3} \pm 120.6 \mu \mathrm{m}^{3}$ (mean $\pm \mathrm{SD}$ ) with a minimal volume of $335 \mu \mathrm{m}^{3}$ [27]. Thus, the volume of the MMCs is beyond the largest cell volumes observed for other cells (Fig. 5C). We therefore eliminated the MMCs from this analysis to eliminate skewing of the results due to their out-of-range size. We observed that the average volume of L1 cells slightly increased during development while the average volume of L2/L3 cells stayed about constant (Fig. 5D) (Table 1). The results further indicated that with the exception of the MMCs the L2 and L3 feature cells of about similar cell volumes while the L1 is composed of smaller cells.

We then compared cell numbers between stages (Fig. 5E) and radial layers (Fig. 5F) (Table 1). Overall, we observed a steady increase in cell numbers during primordium outgrowth (Fig. 5E). The L1 covers a larger surface of the primordium than the L2 or L3.

324 Considering this aspect and given the smaller cell volume of L1 cells in comparison to 325 L2/L3 cells, we hypothesized that the L1 consists of more cells than the L2 and L3. This assumption was supported by layer-specific cell counts (Fig. 5F) (Table 1). We also determined that the L1 showed the largest percentage increase in cell numbers while the L3 showed the least percentage increase in cell numbers (Fig. 5G). 
Next, we assessed the spatial distribution of mitoses. To this end we manually labelled cells that exhibit mitotic figures (Fig. 5A,B). We identified a total of 52 mitotic cells in our dataset comprising 52 3D digital ovule primordia. We first asked if there were differences in the number of mitoses between the cell layers. We found 33 mitotic cells in the L1, 18 in the L2, and 1 in the L3. This result is in line with the observed differences in cell numbers between the three layers (Fig. 5F). We then investigated if there was a difference between the number of mitotic cells in the anterior and posterior L1. We found 23 and 10 mitoses in the anterior and posterior L1, respectively, indicating that more cell divisions occur in the anterior domain. Finally, we analysed the proximal-distal distribution of mitoses. We found that about 80 to 85 percent of scored mitotic cells were located in the proximal half of the developing primordium (Fig. $5 \mathrm{H}$ ).

Taken together, our data indicate that primordium outgrowth is preferentially driven by an increase in cell number, not cell volume. In addition, they suggest unequal spatial

344 distribution of mitoses between cell layers and along the anterior-posterior and proximal345 distal axes. A higher number of mitoses in the anterior domain might explain primordium slanting. The data further indicate that a cell proliferation zone located in the bottom half

347 of the developing primordium contributes significantly to its outgrowth.

\section{Funiculus curvature correlates with differences in cell number}

\section{and cell volume along the anterior-posterior and proximal-}

\section{distal axes}

352 To obtain insight into the cellular processes underlying funiculus curvature we performed 
(Fig. 6A-E). By this stage growth of the funiculus has ceased [27]. We focused on the L1 along the L1 midlines of the anterior and posterior domains, respectively (Fig. 6A-E). We observed that the anterior midline was longer and characterized by a higher number of cells in comparison to the posterior midline. To directly compare volumes of anterior and posterior cells we converted the cells' coordinates into relative proximal-distal positions [53]. There we noticed a gradient in the volume of anterior L1 and L2 cells with the distal-most cells featuring nearly 1.5 to 2 times the volume of cells located at the proximal end (Fig. 6D,E). We did not detect a noticeable volume increase in the posterior cells.

In summary, our data suggest that a combination of differential cell proliferation along the anterior-posterior axis and unequal cell growth along the proximal-distal axis of the anterior domain contributes to the curvature of the funiculus.

\section{Proximal-distal growth gradient in Arabidopsis integuments}

371 Genetic data indicated that asymmetric growth of the outer integument contributes

372 significantly to the anatropous shape of the ovule $[27,54,55]$. However, the 3D architecture of integument cells in relation to their position within the tissue remained unknown. Thus, we undertook a first analysis of the 3D geometry of integument cells in 3D digital ovules of stage 3-IV. At this stage curvature is underway but not yet completed. We used 3DCoordX to measure cell volumes of the outer layer of the outer integument along the proximal-distal axis. We found a gradient in cell volume along this axis. We observed that proximal cells exhibited small cell volumes while, with the 
exception of small cells at the tip of the integument, distal cells were characterized by larger sizes (Fig. 7A). Next, we expanded the 3D cellular analysis to all cells of the integuments. In a typical stage 3-IV 3D digital ovule we found a proximal-distal gradient of cell volumes in cells of the other layers, but at a smaller scale compared to the outer layer of the outer integument (Fig. 7A-C). We then compared cell length to cell position along the proximal-distal axis in the medial domain of the inner layer of the outer integument across five different specimens. We found that cell length increased along the proximal-distal axis (Fig. 7D).

Taken together, our data suggest that preferential cell elongation along the proximal-distal axis may be an important factor underlying differential growth of the outer integument and ovule curvature.

\section{Application to other plant organs}

Finally, we explored if our approach for an organ-intrinsic coordinate system was useful growth patterns in different organs of various plant species. To this end we investigated 3D digital plant organs of diverse morphological complexity. We first inspected the archegonium of the liverwort genetic model system Marchantia polymorpha. The archegonium is an organ of simple morphology consisting of two main tissues: the spherical venter harboring the egg cell and the elongated neck through which the sperm cell reaches the egg cell [56]. We generated two 3D digital archegonia: a younger specimen A and an older specimen B. Both archegonia were imaged, 3D segmented, and

402 cells of the neck, neck canal, venter, and venter canal were identified and labelled manually. We removed the egg and canal cells from our analysis and focused on the 
404

405

406

407

408

409

410

411

412

413

414

415

416

417

418

419

420

421

422

423

424

425

426

427

428

venters and the necks of the two 3D digital archegonia (Fig. 8A,B). A first inspection already revealed differences between the two specimens. We observed that the venter of specimen A possessed only one cell layer with no obvious signs of periclinal cell division. By contrast, we found that a scattered pattern of periclinal cell divisions had occurred in the venter of specimen B associated with the formation of a second cell layer (Fig. 8B) [56]. This observation indicated temporal and spatial asynchrony in the control of these periclinal cell divisions.

A monolayer of cells formed the necks of both specimens. We implemented an organ coordinate system to enable a spatial analysis of some basic cellular parameters along the long axes of the two specimens. We placed a Bezier ring at the boundary between the venter and neck cells (Fig. 8A,B). The placement of the ring resulted in the assignment of positive and negative organ coordinate values for the venter and neck cells, respectively.

We then assessed the basis of the differences in neck lengths between the two specimens. Measuring neck length along the main central axis revealed that the neck of specimen B was about 2 times longer than specimen A (329 $\mu \mathrm{m}$ versus $160 \mu \mathrm{m})$. We then asked if the length disparity between the necks of the two specimens was due to a difference in cell numbers and/or cell elongation. We determined 98 and 114 neck cells for specimens A and $\mathrm{B}$, respectively, indicating a minor difference in neck cell numbers. Next, we quantified cell length along the central organ axis for all neck cells. We observed that the neck cells of specimen A had an average length of $10.3 \mu \mathrm{m} \pm 2.4 \mu \mathrm{m}$ and exhibited a relatively uniform cell length (Fig. 8C). Neck cells of specimen B showed a more heterogeneous distribution of cell length and were noticeably more elongated with an average cell length of $20.8 \pm 5.6 \mu \mathrm{m}$. Moreover, cell elongation increased towards the tip of the neck in specimen B while no such increase was observed for specimen A (Fig. 8D). 
The results indicated that enhanced cell elongation along the central axis of the neck was mainly associated with the increase in neck length in specimen B in comparison to specimen A.

Finally, we turned our attention to a plant organ of complex 3D morphology. The cupshaped trap of the aquatic carnivorous plant Utricularia gibba represents a highly curved 3D leaf form $[57,58]$. Quantitative growth analysis at the tissue level combined with computer modeling indicated that the complex shape transformations occurring during trap development are associated with differential rates and orientations of growth $[59,60]$. However, a quantitative analysis of 3D cellular parameters had not been performed. To obtain first insight into the cellular basis of the growth patterns shaping the Utricularia trap we generated a 3D digital representation with cellular resolution of an intermediatestage trap collected 6 days after initiation [59]. By this stage an invagination in the nearspherical young trap had occurred, followed by the formation of further folds and tissue broadening, and resulting in the emergence of the interior trap door and threshold (Fig. 9A-C). We manually labelled the various tissues, including the abaxial and adaxial cells of the wall, the threshold, and the combined trap door/palate domain, and distinguished between medial and lateral domains of the adaxial and abaxial wall, respectively. To define an origin of the distance coordinate system we placed an ellipsoid Bezier ring at the boundary between the base of the threshold and the wall of the trap (Fig. 9D). We then asked if there were position-related differences in cell volume in the epidermal layers of the wall and threshold by analyzing epidermal cells located along the respective midlines of the tissues (Fig. 9E-G). We observed that cell volume varied along the measured distances. For example, we noticed a sudden increase in cell volume in an interval from $240 \mu \mathrm{m}$ to $320 \mu \mathrm{m}$ for cells of the abaxial wall (Fig. 9F). This region 
precedes a prominent kink in the abaxial wall (Fig. 9E). By contrast, cell volumes of the adaxial wall dropped towards the end. The decrease in cell volume was likely associated with the tapering of the adaxial wall that could be observed in this area. Volumes of threshold cells positioned within a range of 80 to about $150 \mu \mathrm{m}$ from the origin showed relatively small volumes in comparison to the cells flanking this interval (Fig. 9G). The $80-150 \mu \mathrm{m}$ zone corresponded to a section of the threshold which was only moderately curved and provided a large surface exposed to the lumen of the trap (Fig. 9E). Taken together, the data revealed spatial differences in cell volume for all three examined tissues of this specimen and support the notion that differential cell growth contributes to the morphogenesis of the Utricularia trap.

\section{Discussion}

The generation of biological form can be explained in terms of growth oriented relative to an organ-centric coordinate system $[7,28,61]$. To understand tissue morphogenesis it is therefore essential to provide spatial context to the quantitative analysis of the molecular and cellular networks that underlie the development of tissues and organs. It requires robust methods that enable the objective assignment of position to individual cells in a rapid and reliable manner. Here, we developed 3DCoordX, a collection of computational tools that enable the assignment of organ-centric coordinate systems to several different plant organs with complex shapes that were not accessible with previous methods. By applying mathematically defined criteria for the annotation of cell position in $3 \mathrm{D}$ the tools largely eliminate user-derived ambiguities in the cell annotation process. 3DCoordX is implemented as an add-on to the open-source software MorphoGraphX [11,14] (preprint). A detailed user guide can be found in the supplement. 3DCoordX enables quantitative 
analyses of cellular features in their spatial context, in a rapid manner, and on a large scale.

482 We took advantage of the recently made available 3D digital reference atlas of the

483 Arabidopsis ovule to develop generic conceptual and computational approaches that enable the assignment of 3D coordinate systems to organs of simple as well as complex curved morphology. Previous efforts, such as iRoCS [9] and 3DCellAtlas [10], relied on externally imposed coordinate systems. The design of the strategies for the annotation of cell position presented here is guided by intrinsic patterning processes and assign cell distance in relation to organ-centric developmental axes. Such tissue polarity axes are thought to play a central role in the spatial control of growth $[28,62,63]$. To accommodate the particular architecture of the ovule we devised two 3D coordinate systems, one for the main "trunk", the central proximal-distal axis consisting of the nucellus, chalaza and funiculus, and one for the integuments. Both coordinate systems are based on similar general principles. First, we distinguish between individual cell layers as in the L1 to L3 layers of the primordium or the adaxial-abaxial (dorso-ventral) cell layers of the two integuments. Importantly, with the concept of "outside wall area ratio" 3DCoordX embodies a new strategy for the classification of the radial layers. It does not rely on a surface mesh for classification and thus is more versatile than other published methods.

498 Second, the cell layers then become subdivided into two domains: the anterior-posterior 499 domains of the trunk and the medial-lateral domains of the integument cell layers. Third, subsequent assignment of a proximal-distal distance value to each cell is constricted by these two prepatterns. For the placement of reference Bezier rings we took cues from the

502 localization of important developmental regulators, such as the presence of an auxin 503 maximum at the distal tip of the ovule primordium or the $C U C 3$ expression in the chalaza 
[39-41,52]. As a result of the approach each cell is annotated in 3D with respect to the

505

506

507

508

509

510

511

512

513

514

515

516

517

518

519

520

521

522

523

524

525

526

527

528

radial and proximal-distal dimensions as well as to either an anterior-posterior or medial-

lateral axis. With the help of 3DCoordX we readily discovered previously unidentified

cellular growth patterns in the primordium and integuments. For example, our data

indicate a basal cell proliferation zone in the ovule primordium and suggest that

preferential cell proliferation in the anterior domain is important for primordium slanting.

Moreover, we obtained evidence that the increase in cell volume along the proximal-

distal axis of the outer integument, a tissue without radial symmetry, is mainly explained by an increase in cell length.

Importantly, our work revealed that the respective principles can be successfully applied to the establishment of coordinate systems for organs of varying degrees of morphological complexity and the subsequent quantitative analysis of $3 \mathrm{D}$ cellular parameters. We provided evidence for a preferential increase in cell length during axial neck growth of the Marchantia archegonium. Moreover, we identified distinct cellular patterns possibly associated with important morphological features of the intricately folded $U$. gibba trap. These data reveal that 3DCoordX has broad applicability and eliminates the need to work with multiple different pipelines when analyzing the cellular architecture of organs in 3D. The general strategies and computational methods put forward in this work will greatly reduce the time required for the spatial analysis of cellular parameters so central to various approaches, such as computational modeling of morphogenesis or comparative morphometry of specimens from different genotypes. 


\section{Plant work and lines}

530 Arabidopsis thaliana (L.) Heynh. var. Columbia (Col-0) was used as a wild-type strain.

531 Plants were grown on soil as described earlier [64]. The pKAN1::KAN1:2xGFP construct

$532[38]$ and the pCUC3::CFP line [52] were gifts from Marcus Heisler and Nicolas Arnaud, transferred to soil for further inspection. Marchantia polymorpha of the BoGa ecotype was grown on half-strength Gamborg's B5 medium under long day conditions (16L:8D) after 4-6 weeks.

\section{Clearing and staining of tissue samples}

544 Treatment of ovules of the pKAN1::KAN1:2xGFP and the pCUC3::CFP lines was done

545 as described in [68] and [27]. Tissue was fixed in 4\% paraformaldehyde in PBS followed

546 by two washes in PBS before transfer into the ClearSee solution (xylitol $(10 \%$, w/v),

547 sodium deoxycholate $(15 \%, w / v)$, urea $(25 \%, w / v)$, in $\mathrm{H} 2 \mathrm{O})$ [69]. Clearing was done at

548 least overnight or for up to two to three days. Cell wall staining with SR2200

549 (Renaissance Chemicals, Selby, UK) was performed as described in [70]. Cleared tissue

550 was washed in PBS and then put into a PBS solution containing 0.1\% SR2200 and a

551 1/1000 dilution of the nuclear stain TO-PRO-3 iodide (Thermo Fisher Scientific) for 20

552 minutes. Tissue was washed in PBS for one minute, transferred again to ClearSee for 20

553 minutes before mounting in Vectashield antifade agent (Vector Laboratories, Burlingame, 
554 CA, USA). Marchantia archegoniophores were fixed for 1 week in 4\% paraformaldehyde in PBS followed by two washes in PBS before transfer to ClearSee. Clearing was done for 4-7 days. Cell wall staining and subsequent clearing, washing and mounting steps were the same as for Arabidopsis ovules. Archegonia were dissected in Vectashield mounting medium (Vector Laboratories, Burlingame, CA, USA).

\section{Microscopy and image acquisition}

561 Confocal laser scanning microscopy of ovules stained with SR2200 and TO-PRO-3

562 iodide was performed on an upright Leica TCS SP8 X WLL2 HyVolution 2 (Leica Microsystems) equipped with GaAsP (HyD) detectors and a 63x glycerol objective (HC PL anterior-posteriorO CS2 63x/1.30 GLYC, CORR CS2). Scan speed was at $400 \mathrm{~Hz}$, the pinhole was set to 0.6 Airy units, line average between 2 and 4, and the digital zoom between 1 and 2. For z-stacks, 12-bit images were captured at a slice interval of $0.24 \mu \mathrm{m}$ with voxel size of $0.125 \mu \mathrm{m} \times 0.125 \mu \mathrm{m} \times 0.24 \mu \mathrm{m}$. Laser power or gain was adjusted for z compensation to obtain an optimal z-stack. Images were adjusted for color and contrast using Adobe Photoshop 2021 (Adobe, San Jose, USA) or MorphographX [14] software. Image acquisition parameters for the pKAN1::KAN1:2xGFP line were the following: SR2200; 405 diode laser 0.10\%, HyD 420-480 nm, detector gain 10. 2xGFP; $488 \mathrm{~nm}$

572 Argon laser 2\%, HyD 525-550 nm, detector gain 100. TO-PRO-3; $642 \mathrm{~nm}$ White Laser $5732 \%$, HyD 660-720 nm, detector gain 100. In each case sequential scanning was 574 performed to avoid crosstalk between the spectra. Image acquisition parameters for the 575 pCUC3::CFP line were the following: SR2200; 405 diode laser 0.10\%, HyD 420-480 $576 \mathrm{~nm}$, detector gain 10. CFP; $514 \mathrm{~nm}$ Argon laser 2\%, HyD 525-550 nm, detector gain 100. 577 TO-PRO-3; $642 \mathrm{~nm}$ White Laser 2\%, HyD 660-720 nm, detector gain 100. In each case sequential scanning was performed to avoid crosstalk between the spectra. Imaging 
conditions for the Marchantia archegonia stained with SR2200 and TO-PRO-3 iodide archegonium was imaged without tile scan.

\section{Datasets and 3D cell segmentation}

591 The dataset encompassing the segmented wild-type 3D digital ovules was described

592 earlier [27]. The two z-stacks of Marchantia archegonia were 3D cell segmented using the PlantSeg pipeline [17]. The z-stack of the Utricularia gibba trap was obtained from a fixed and modified pseudo-Schiff-stained [71] specimen [59]. 3D cell segmentation was performed using the PlantSeg-MorphoGraphX hybrid method as described in [27]. In all instances generation of cell surface meshes and cell type labeling was performed with MorphoGraphX.

\section{Software}

600 The MorphographX software was used for the generation of cell surface meshes, cell type labeling, and the analysis of 3D cellular features [14]. It can be downloaded from its website (https://www.mpiz.mpg.de/MorphoGraphX). The 3DCoordX toolbox is 
604

605

606

607

608

609

610

611

612

613

614

615

616

617

618

619

620

621

622

623

\section{Authors' contributions}

625 AV, SS, RS and KS designed the study. AV, SS, RT, TAM, and KL performed the 626

supplement. The PlantSeg pipeline [17] was used for 3D cell boundary prediction and segmentation. The software can be obtained from its Github repository (https://github.com/hci-unihd/plant-seg).

\section{Acknowledgements}

We thank Marcus Heisler and Nicolas Arnaud for the pKAN1::KAN1:2xGFP and pCUC3::CFP lines, respectively. We also thank Peter Schroeder and Claus

Schwechheimer for providing Marchantia polymorpha female gametophyte samples. We thank members of the Schneitz lab for helpful discussions and Adam Runions for discussions on growth alignment graphs. We further thank Enrico Coen for insightful comments. We acknowledge support by the Center for Advanced Light Microscopy (CALM) of the TUM School of Life Sciences. This work was funded by the German Research Council (DFG) through grants FOR2581 (TP7) to KS, (TP8) to RS, (TP9) to MT and a Max Planck Society core grant to MT. Work by KL was funded by the Biotechnology and Biological Sciences Council (BBSRC) through grants BBS/E/J/00000152, BBS/E/J/000PR9787, and BBS/E/J/000CA517.

\section{Competing interests}

There are no financial or non-financial competing interests.
experiments. AV, SS, RT, TAM, KL, MT, RS and KS interpreted the results. MT, RS and 
KS secured funding. KS wrote the paper with comments from all authors. All authors read and approved the final manuscript.

629

\section{References} screening. Cell. 2015;163: 1314-1325. Heterogeneity and robustness in plant morphogenesis: from cells to organs. Annu Rev Plant Biol. 2018;69: 469-495. Landrein B, et al. Global Topological Order Emerges through Local Mechanical Control of Cell Divisions in the Arabidopsis Shoot Apical Meristem. Cell Syst. 2019;8: 53-65.e3.

4. Kierzkowski D, Runions A, Vuolo F, Strauss S, Lymbouridou R, RoutierKierzkowska A-L, et al. A Growth-Based Framework for Leaf Shape Development and Diversity. Cell. 2019;177: 1405-1418.e17.

5. Kierzkowski D, Routier-Kierzkowska A-L. Cellular basis of growth in plants: geometry matters. Curr Opin Plant Biol. 2019;47: 56-63. epidermal cell shape regulation: different pieces of the same puzzle. Curr Opin Plant Biol. 2019;47: 1-8. Cambridge University Press; 1942. coordinate system in growing plant organ. Acta Soc Bot Pol. 1984;53: 29-42. al. The iRoCS Toolbox--3D analysis of the plant root apical meristem at cellular resolution. Plant J. 2014;77: 806-814.

10. Montenegro-Johnson TD, Stamm P, Strauss S, Topham AT, Tsagris M, Wood ATA, et al. Digital Single-Cell Analysis of Plant Organ Development Using 3DCellAtlas. Plant Cell. 2015;27: 1018-1033. 
12. Fernandez R, Das P, Mirabet V, Moscardi E, Traas J, Verdeil J-L, et al. Imaging plant growth in 4D: robust tissue reconstruction and lineaging at cell resolution. Nat Methods. 2010;7: 547-553.

13. Lowekamp BC, Chen DT, Ibáñez L, Blezek D. The design of SimpleITK. Front Neuroinform. 2013;7: 45.

14. Barbier de Reuille P, Routier-Kierzkowska A-L, Kierzkowski D, Bassel GW, Schüpbach T, Tauriello G, et al. MorphoGraphX: A platform for quantifying morphogenesis in 4D. Elife. 2015;4: 05864.

15. Stegmaier J, Amat F, Lemon WC, McDole K, Wan Y, Teodoro G, et al. Real-time three-dimensional cell segmentation in large-scale microscopy data of developing embryos. Dev Cell. 2016;36: 225-240.

16. Eschweiler D, Spina TV, Choudhury RC, Meyerowitz E, Cunha A, Stegmaier J. CNN-based preprocessing to optimize watershed-based cell segmentation in 3D confocal microscopy images. IEEE; 2019. pp. 223-227.

17. Wolny A, Cerrone L, Vijayan A, Tofanelli R, Barro AV, Louveaux M, et al. Accurate and versatile 3D segmentation of plant tissues at cellular resolution. Elife. 2020;9. doi:10.7554/eLife.57613

18. Bassel GW, Stamm P, Mosca G, Barbier de Reuille P, Gibbs DJ, Winter $\mathrm{R}$, et al. Mechanical constraints imposed by 3D cellular geometry and arrangement modulate growth patterns in the Arabidopsis embryo. Proc Natl Acad Sci U S A. 2014;111: 8685-8690.

19. Yoshida S, Barbier de Reuille P, Lane B, Bassel GW, Prusinkiewicz P, Smith RS, et al. Genetic control of plant development by overriding a geometric division rule. Dev Cell. 2014;29: 75-87.

20. Lora J, Herrero M, Tucker MR, Hormaza JI. The transition from somatic to germline identity shows conserved and specialized features during angiosperm evolution. New Phytol. 2017;216: 495-509.

21. Pasternak T, Haser T, Falk T, Ronneberger O, Palme K, Otten L. A 3D digital atlas of the Nicotiana tabacum root tip and its use to investigate changes in the root apical meristem induced by the Agrobacterium 6b oncogene. Plant J. 2017;92: 31-42.

22. Montenegro-Johnson T, Strauss S, Jackson MDB, Walker L, Smith RS, Bassel GW. 3DCellAtlas Meristem: a tool for the global cellular annotation of shoot apical meristems. Plant Methods. 2019;15: 33.

23. Graeff M, Rana S, Wendrich JR, Dorier J, Eekhout T, Fandino ACA, et al. A morpho-transcriptomic map of brassinosteroid action in the Arabidopsis root. Molecular Plant. 2021; 2021.03.30.437656.

24. Hernandez-Lagana E, Mosca G, Mendocilla-Sato E, Pires N, Frey A, Giraldo-Fonseca A, et al. Organ geometry channels reproductive cell fate in the Arabidopsis ovule primordium. Elife. 2021;10. doi:10.7554/eLife.66031 
25. Refahi Y, Zardilis A, Michelin G, Wightman R, Leggio B, Legrand J, et al. A multiscale analysis of early flower development in Arabidopsis provides an integrated view of molecular regulation and growth control. Dev Cell. 2021;56: 540556.e8.

26. Silveira SR, Le Gloanec C, Gómez-Felipe A, Routier-Kierzkowska A-L, Kierzkowski D. Live-imaging provides an atlas of cellular growth dynamics in the stamen. Plant Physiol. 2021 [cited 5 Aug 2021]. doi:10.1093/plphys/kiab363

708

709

710

711

712

713

714

715

716

717

718

719

720

721

722

723

724

725

726

27. Vijayan A, Tofanelli R, Strauss S, Cerrone L, Wolny A, Strohmeier J, et al. A digital 3D reference atlas reveals cellular growth patterns shaping the Arabidopsis ovule. Elife. 2021;10. doi:10.7554/eLife.63262

28. Whitewoods CD, Coen E. Growth and development of three-dimensional plant form. Curr Biol. 2017;27: R910-R918.

29. Bouman F. The ovule. Johri BM, editor. Embryology of Angiosperms. New York: Springer Verlag; 1984. pp. 123-157.

30. Endress PK. Angiosperm ovules: diversity, development, evolution. Ann Bot. 2011;107: 1465-1489.

31. Robinson-Beers K, Pruitt RE, Gasser CS. Ovule development in wild-type Arabidopsis and two female-sterile mutants. Plant Cell. 1992;4: 1237-1249.

32. Schneitz K, Hülskamp M, Pruitt RE. Wild-type ovule development in Arabidopsis thaliana: a light microscope study of cleared whole-mount tissue. Plant J. 1995; 7: 731-749.

33. Jenik PD, Irish VF. Regulation of cell proliferation patterns by homeotic genes during Arabidopsis floral development. Development. 2000;127: 1267-1276.

34. Satina S, Blakeslee AF, Avery AG. Demonstration of the three germ layers in the shoot apex of Datura by means of induced polyploidy in periclinal chimeras. Am J Bot. 1940;27: 895-905.

35. Reiser L, Modrusan Z, Margossian L, Samach A, Ohad N, Haughn GW, et al. The BELL1 gene encodes a homeodomain protein involved in pattern formation in the Arabidopsis ovule primordium. Cell. 1995;83: 735-742.

36. Gross-Hardt R, Lenhard M, Laux T. WUSCHEL signaling functions in interregional communication during Arabidopsis ovule development. Genes Dev. 2002;16: 1129-1138.

37. Sieber P, Gheyselinck J, Gross-Hardt R, Laux T, Grossniklaus U, Schneitz K. Pattern formation during early ovule development in Arabidopsis thaliana. Dev Biol. 2004;273: 321-334.

38. Caggiano MP, Yu X, Bhatia N, Larsson A, Ram H, Ohno CK, et al. Cell type boundaries organize plant development. Elife. 2017;6. doi:10.7554/eLife.27421

39. Benková E, Michniewicz M, Sauer M, Teichmann T, Seifertová D, 
Jürgens $\mathrm{G}$, et al. Local, efflux-dependent auxin gradients as a common module for plant organ formation. Cell. 2003;115: 591-602. Reporters for sensitive and quantitative measurement of auxin response. Nat Methods. 2015;12: 207-10, 2 p following 210.

41. Kawamoto N, Del Carpio DP, Hofmann A, Mizuta Y, Kurihara D, Higashiyama $\mathrm{T}$, et al. A peptide pair coordinates regular ovule initiation patterns with seed number and fruit size. Curr Biol. 2020;30: 4352-4361.e4. factors BEL1 and SPL are required for cytokinin and auxin signaling during ovule development in Arabidopsis. Plant Cell. 2012;24: 2886-2897.

43. Galbiati F, Sinha Roy D, Simonini S, Cucinotta M, Ceccato L, Cuesta C, et al. An integrative model of the control of ovule primordia formation. Plant J. 2013;76: 446-455. Genes Dev. 1999;13: 3160-3169.

45. McAbee JM, Hill TA, Skinner DJ, Izhaki A, Hauser BA, Meister RJ, et al. ABERRANT TESTA SHAPE encodes a KANADI family member, linking polarity determination to separation and growth of Arabidopsis ovule integuments. Plant J. 2006;46: 522-531.

46. Kelley DR, Skinner DJ, Gasser CS. Roles of polarity determinants in ovule development. Plant J. 2009;57: 1054-1064.

47. Aida M, Ishida T, Fukaki H, Fujisawa H, Tasaka M. Genes involved in organ separation in Arabidopsis: an analysis of the cup-shaped cotyledon mutant. Plant Cell. 1997;9: 841-857.

48. Ishida T, Aida M, Takada S, Tasaka M. Involvement of CUP-SHAPED COTYLEDON genes in gynoecium and ovule development in Arabidopsis thaliana. Plant Cell Physiol. 2000;41: 60-67. COTYLEDON1 gene of Arabidopsis regulates shoot apical meristem formation. Development. 2001;128: 1127-1135.

50. Breuil-Broyer S, Morel P, de Almeida-Engler J, Coustham V, Negrutiu I, Trehin C. High-resolution boundary analysis during Arabidopsis thaliana flower development. Plant Journal. 2004;38: 182-192.

51. Sieber P, Wellmer F, Gheyselinck J, Riechmann JL, Meyerowitz EM. Redundancy and specialization among plant microRNAs: role of the MIR164 family in developmental robustness. Development. 2007;134: 1051-1060.

52. Gonçalves B, Hasson A, Belcram K, Cortizo M, Morin H, Nikovics K, et 

development. Plant J. 2015;83: 732-742. $\mathrm{B}$, et al. A WOX/auxin biosynthesis module controls growth to shape leaf form. Curr Biol. 2020. doi:10.1016/j.cub.2020.09.037

54. Baker SC, Robinson-Beers K, Villanueva JM, Gaiser JC, Gasser CS. Interactions among genes regulating ovule development in. Genetics. 1997;145: 1109-1124.

55. Schneitz K, Hülskamp M, Kopczak SD, Pruitt RE. Dissection of sexual organ ontogenesis: a genetic analysis of ovule development in Arabidopsis thaliana. Development. 1997;124: 1367-1376.

789

790

791

792

793

794

795

796

797

798

799

800

801

802

803

804

805

806

807

808

809

810

811

812

813

56. Shimamura M. Marchantia polymorpha: taxonomy, phylogeny and morphology of a model system. Plant Cell Physiol. 2016;57: 230-256.

57. Płachno BJ, Adamec L, Kamińska I. Relationship between trap anatomy and function in Australian carnivorous bladderworts (Utricularia) of the subgenus Polypompholyx. Aquat Bot. 2015;120: 290-296.

58. Reifenrath K, Theisen I, Schnitzler J, Porembski S, Barthlott W. Trap architecture in carnivorous Utricularia (Lentibulariaceae). Flora - Morphology, Distribution, Functional Ecology of Plants. 2006;201: 597-605.

59. Lee KJI, Bushell C, Koide Y, Fozard JA, Piao C, Yu M, et al. Shaping of a three-dimensional carnivorous trap through modulation of a planar growth mechanism. PLoS Biol. 2019;17: e3000427.

60. Whitewoods CD, Gonçalves B, Cheng J, Cui M, Kennaway R, Lee K, et al. Evolution of carnivorous traps from planar leaves through simple shifts in gene expression. Science. 2020;367: 91-96.

61. Coen E, Rebocho AB. Resolving conflicts: modeling genetic control of plant morphogenesis. Dev Cell. 2016;38: 579-583.

62. Kennaway R, Coen E, Green A, Bangham A. Generation of diverse biological forms through combinatorial interactions between tissue polarity and growth. PLoS Comput Biol. 2011;7: e1002071.

63. Kuhlemeier C, Timmermans MCP. The Sussex signal: insights into leaf dorsiventrality. Development. 2016;143: 3230-3237.

64. Fulton L, Batoux M, Vaddepalli P, Yadav RK, Busch W, Andersen SU, et al. DETORQUEO, QUIRKY, and ZERZAUST represent novel components involved in organ development mediated by the receptor-like kinase STRUBBELIG in Arabidopsis thaliana. PLoS Genet. 2009;5: e1000355.

65. Koncz C, Schell J. The promoter of TL-DNA gene 5 controls the tissuespecific expression of chimaeric genes carried by a novel Agrobacterium binary vector. Mol Gen Genet. 1986;204: 383-396. 


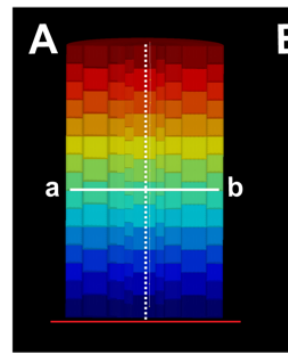

B 71. Truernit E, Bauby H, Dubreucq B, Grandjean O, Runions J, Barthélémy J, et al. High-resolution whole-mount imaging of three-dimensional tissue organization and gene expression enables the study of Phloem development and structure in Arabidopsis. Plant Cell. 2008;20: 1494-1503.

69. Kurihara D, Mizuta Y, Sato Y, Higashiyama T. ClearSee: a rapid optical clearing reagent for whole-plant fluorescence imaging. Development. 2015;142: 4168-4179.

70. Musielak TJ, Schenkel L, Kolb M, Henschen A, Bayer M. A simple and versatile cell wall staining protocol to study plant reproduction. Plant Reprod. 2015;28: 161-169.

\section{Figure legends}

841 Fig 1. Axial cell distance determination in curved tissue. (A) Section through an

842 artificial template of a tube-like and straight tissue consisting of multiple concentric cell

843 layers. The heatmap indicates distance from the reference (red line at bottom). The

844 dashed line outlines the central axis. Note that the two cells $(a, b)$ at the same cell index 
845 position also show the same absolute axial distance to the origin (B) Same structure as in

846 (A) but curved. Note that cells a and b differ in their axial distances to the reference. (C)

847 Same structure as in (B). The separate cell layers are distinguished by their different

848 colors. Two cells in different layers are highlighted (c, b). Dashed lines indicate shortest

849 distances to the reference ignoring tissue layers. Solid lines mark the shortest distances to

850 the reference that are restricted to tissue layers. Confining the shortest distance to a given

851 layer reduces axial distance errors. The red line at the bottom highlights the reference.

852 The arrowhead marks origin cells outlined in red. Origin cells exhibit a close distance in

$8533 \mathrm{D}$ to the reference $(5-15 \mu \mathrm{m})$. (D) $3 \mathrm{D}$ representation of $(\mathrm{C})$ revealing how the anterior-

854 posterior boundary further minimizes the axial distance error for a cell in the posterior

855 half of the structure. 


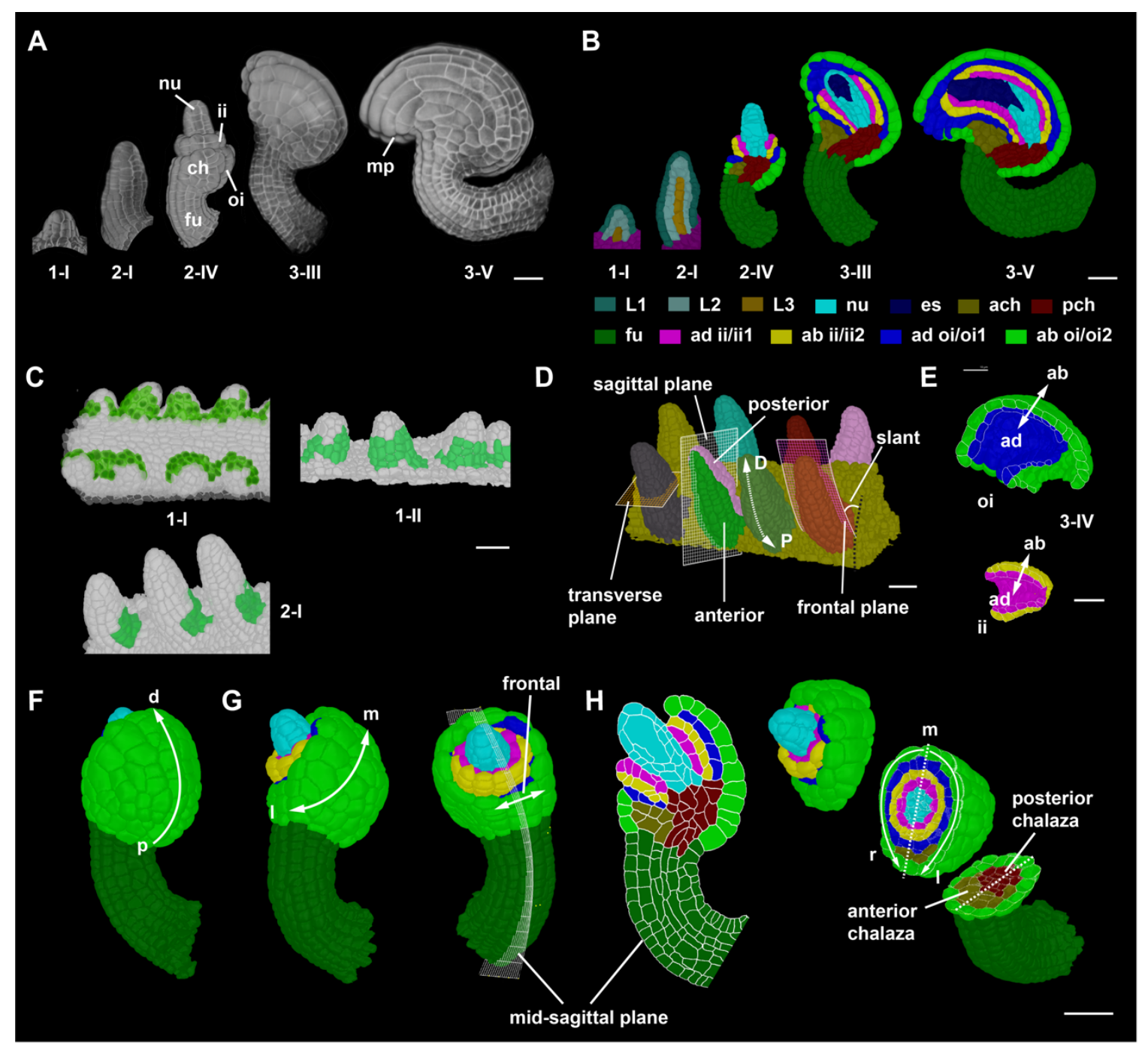

Fig 2. Developmental axes in ovule development. (A) 3D renderings of confocal z-

stacks of SR2200-stained cell walls of wild-type ovules of the indicated stages. (B) Mid-

sagittal sections through 3D digital ovules shown in (A). The different tissues are

863 placenta area carrying eight wild-type stage 2-I ovules. The anterior-posterior and

864 proximal-distal axes are marked. The sagittal, transverse, and frontal planes are marked

865 by grids on the primordia. Frontal plane separates the anterior and posterior halves of the organ. A Sagittal plane separates the left and right half of the organ. The dashed white 
to which the posterior side of the ovule is slanted with a small angle. (E) Outer and inner integument tissues extracted from the 3D mesh for visualizing the abaxial-adaxial

870 polarity. Tilted view of mid-sagittal sections through the outer and inner integument,

871 respectively, of a stage 3-IV 3D digital ovule. The arrows highlight the adaxial-abaxial

872 axes of each integument. (F) Posterior view of a stage 2-V 3D digital ovule with the

873 proximal-distal axis of the outer integument marked. (G) Side view (left) and anterior

874 view (right) of the 3D digital ovule shown in $(\mathrm{F})$. The medial-lateral axis of the outer

875 integument and the frontal region are indicated. (H) A mid-sagittal section view (left) and

876 a 3D clipped view (right) of the 3D digital ovule shown in $(\mathrm{F})$ is depicted. It is oriented

877 with the posterior side to the right. Tissue annotation as in (B). The 3D view allows the

878 discrimination of the left-right sides of the 3D digital ovule. The dashed line indicates the

879 medial line. Abbreviations: ab, abaxial; ad, adaxial; ach, anterior chalaza; ch, chalaza; ii,

880 inner integument; es, embryo sac; fu, funiculus; mp, micropyle; nu, nucellus; oi, outer

881 integument; pch, posterior chalaza; ml, medial-lateral; pd, proximal-distal; rl, right-left.

882 Scale bars: $20 \mu \mathrm{m}$. 

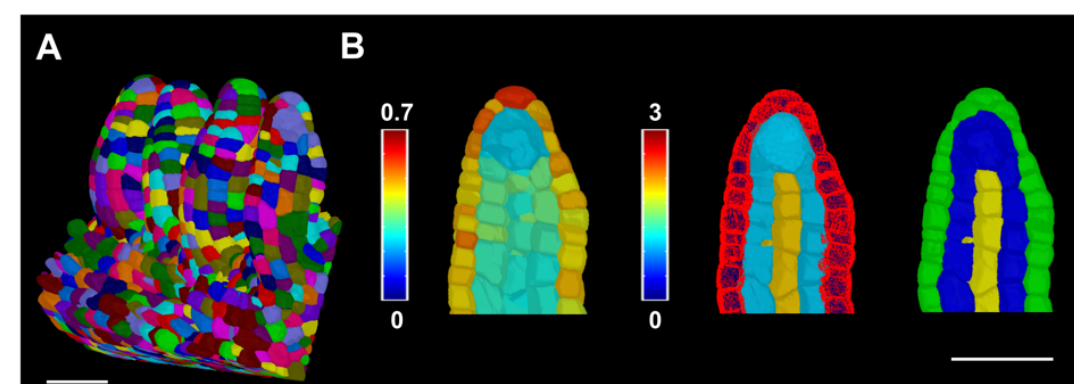

C
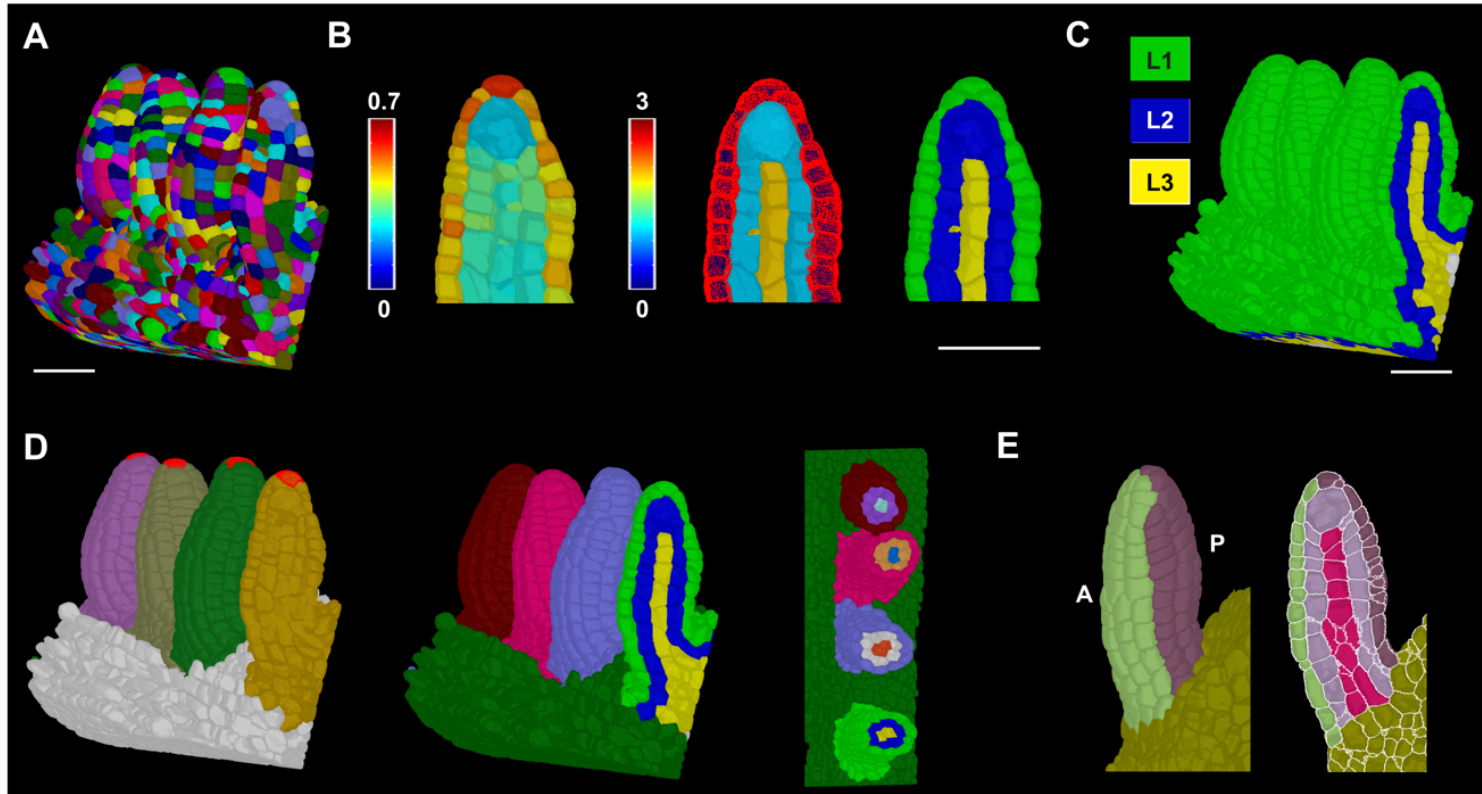

E
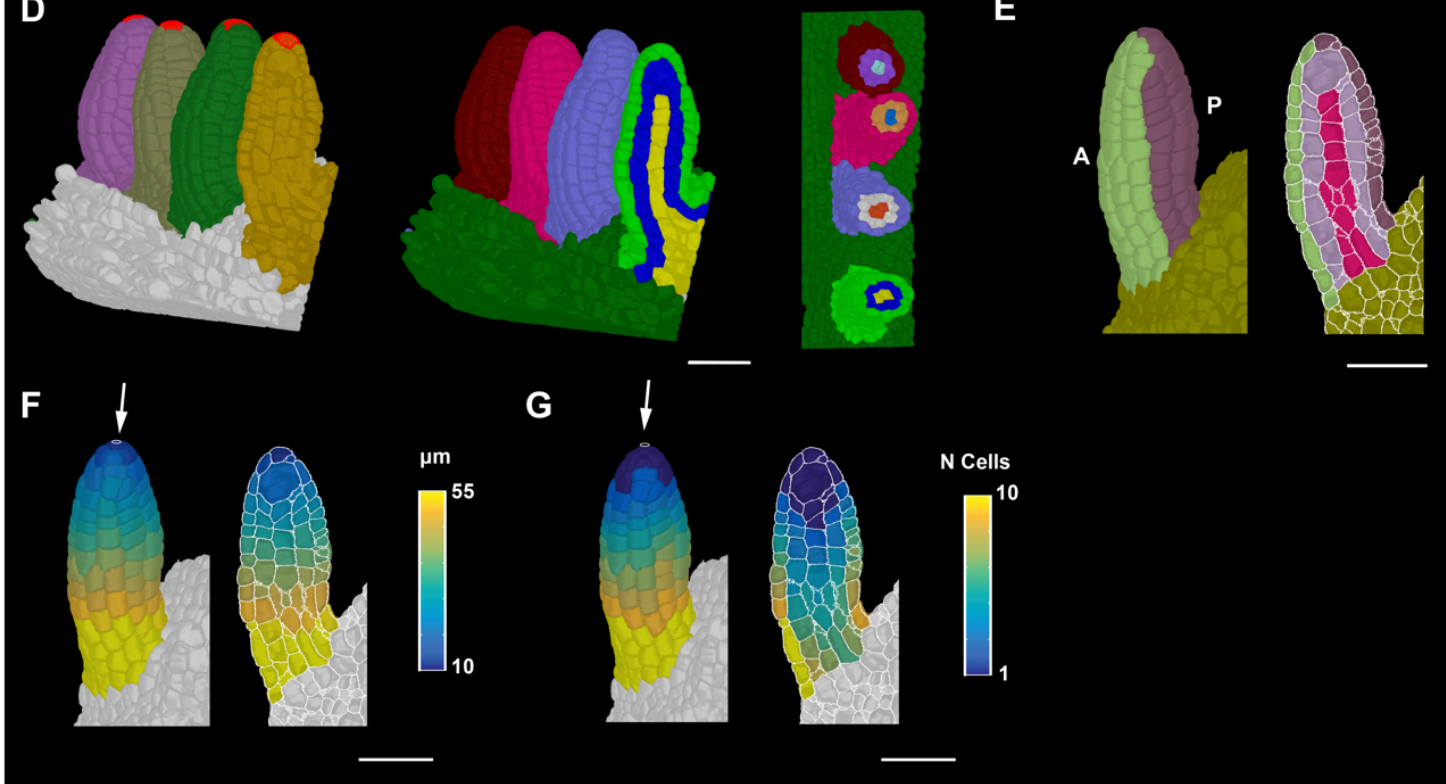

$\mathbf{G} \downarrow$
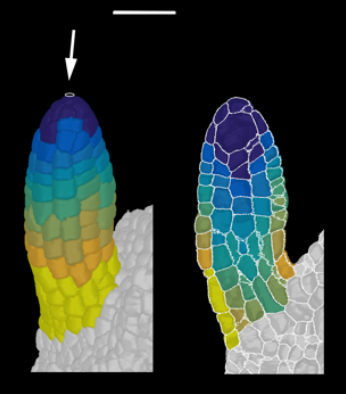

N Cells

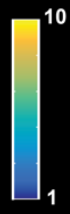

Fig 3. Ovule primordium tissue detection and coordinate system. (A) 3D segmented

cell mesh view of a pistil fragment with four ovules of late stage 2-I. (B) Left panel:

zoomed view of a sagittal section displaying the heatmap of outside wall area ratio.

Threshold selection of outer surface cells based on the heatmap of outside wall area ratio.

Center panel: heatmap indicates cell index, the number of cells an individual cell is

separated from the selected outer surface cells marked in red. Right panel: heatmaps of cell distances (from center panel) were converted to integer values representing the tissue identity labels L1, L2 and L3. (C) Same method applied to the entire 3D mesh shown in (A). (D) Left panel: 3D Mesh view of the specimens shown in (A) with the distal most cell selected for organ separation. Center panel: colors on individual ovule primordia represent the results of organ separation after selecting the distal most cell and clustering the cell connectivity network. Result of the combination of L1, L2 and L3 label and organ 
separated labels annotated for the ovules shown in (A). Right panel: transverse section

898 displaying the L1, L2, L3 labels for different ovules in different colors. (E) Anterior and

899 posterior labels added to the tissue-annotated ovule primordia 3D cell meshes. Left panel:

900 surface view. Right panel: mid-sagittal section. (F) Heatmap of distance coordinates from

901 the point-like origin at the distal end of the organ (white arrow). Heat values indicate the

902 distance in $\mu \mathrm{m}$ from individual cells centroid to the Bezier ring (indicated by white arrow)

903 of the coordinates in a tissue restricted manner. Left panel: 3D view. Right panel: sagittal

904 section view. (G) Heatmap of cell coordinates instead of distance coordinates as in (F).

905 Heat values indicate how many cells apart is a cell of interest from the origin of the

906 coordinates through tissue-restricted manner. Left panel: 3D view. Right panel: sagittal

907 section view. Scale bars: $20 \mu \mathrm{m}$.

908 

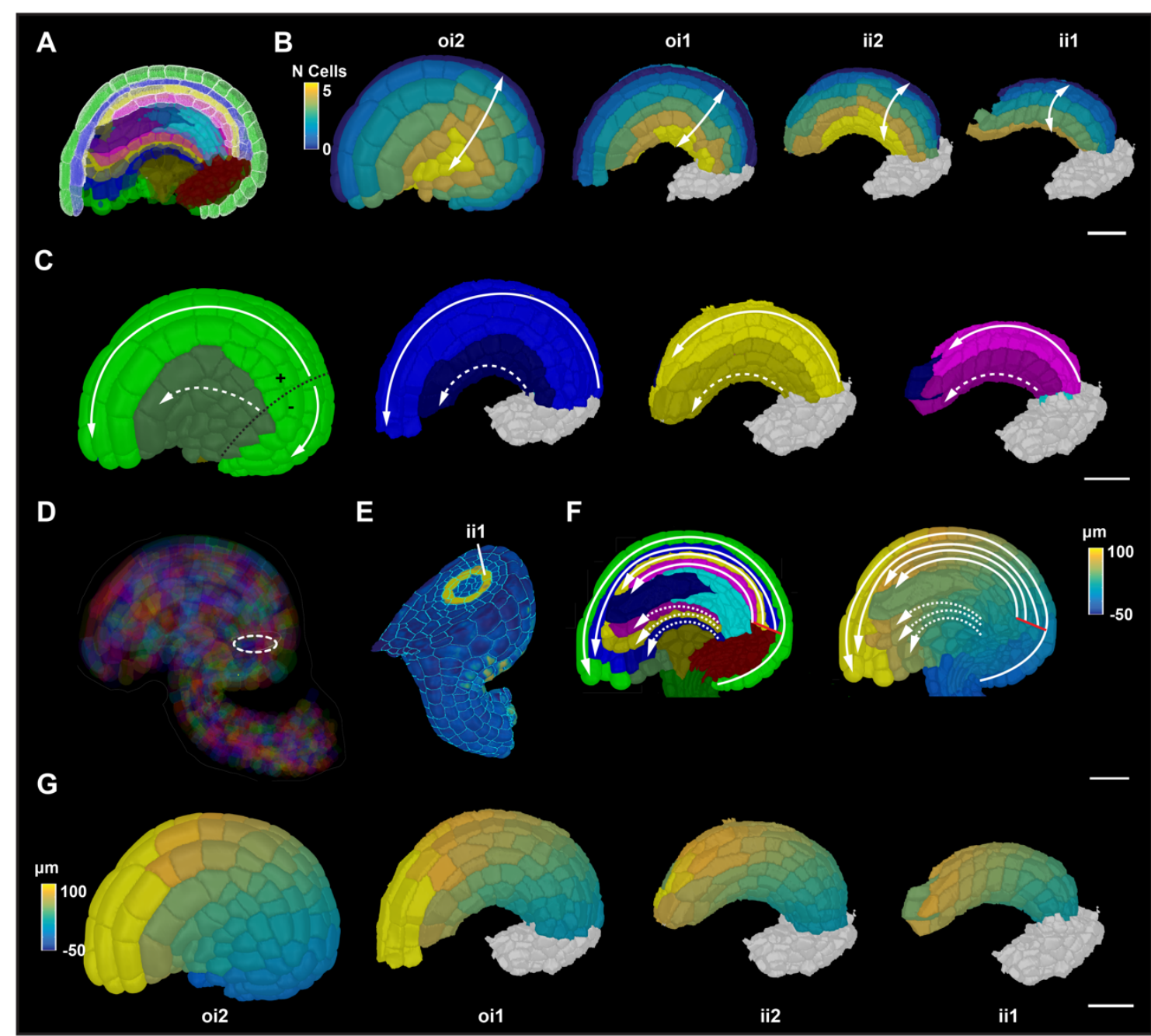

Fig 4. Integument coordinate system. (A) Mid-sagittal section highlighting the selected

911 medial cells on the posterior side of the four layers of integument tissues for medial-

912 lateral coordinate annotation. Colors represent tissue annotations similar to Fig. 1B. (B)

913 Heatmap of medial-lateral cell coordinates. Heat values indicate the lateral position in

914 terms of the number of cells from the median file of cells. Different integument tissues

915 are extracted from the 3D mesh to display the medial-lateral coordinates at their tissue

916 surface. (C) 3D surface view of integument tissues similar to (B). Medial and lateral cells

917 are distinguished. Solid white line represents the tissue restricted coordinate direction

918 along the medial group of cells. White dashed line represents the tissue restricted

919 coordinate direction along the lateral group of cells. Black dotted line on oi2 represents

920 the coordinate origin projected on the surface which separates the proximal oi2 cells with 
negative coordinate values (D) Semi-transparent view of a mature 3D ovule displaying

922 the coordinate origin as a ring inside the organ. (E) 3D clipping view of a transverse

923 section of an ovule highlighting the ring-like expression of the pCUC3::CUC3:CFP

924 reporter in yellow. (F) Left panel: sagittal section of a mature ovule displaying the

925 coordinate directions of the medial and lateral group of cells in solid and white lines,

926 respectively. Solid red line indicates the origin of the coordinate system. Right panel:

927 Sagittal section displaying the heatmap of distance coordinates. Solid red line indicates

928 the origin of the coordinate system. (G) 3D surface view of integument tissues similar to

929 (B) displaying the distance coordinates at the surface of internal tissues. Scale bars: 20

$930 \mu \mathrm{m}$.

931 

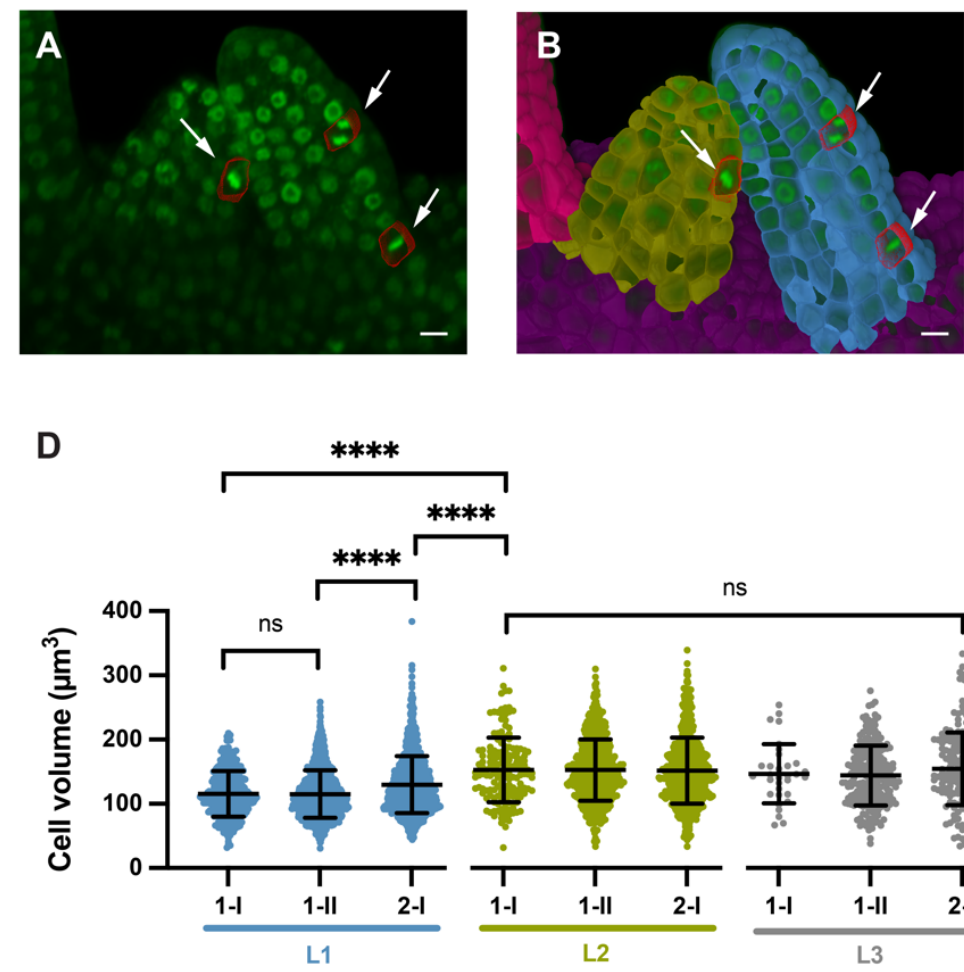

$\mathbf{F}$

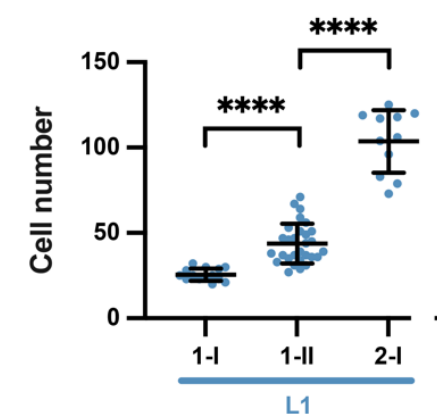

H

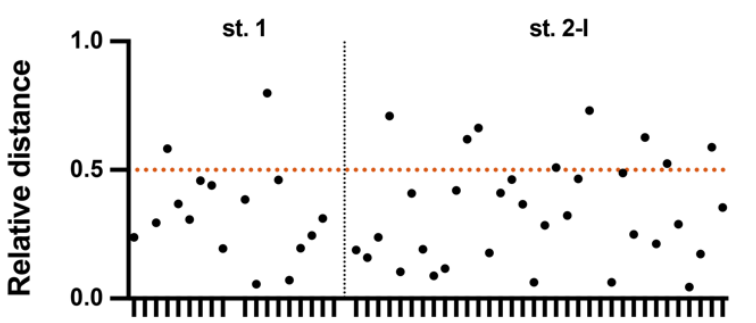

Cell ID
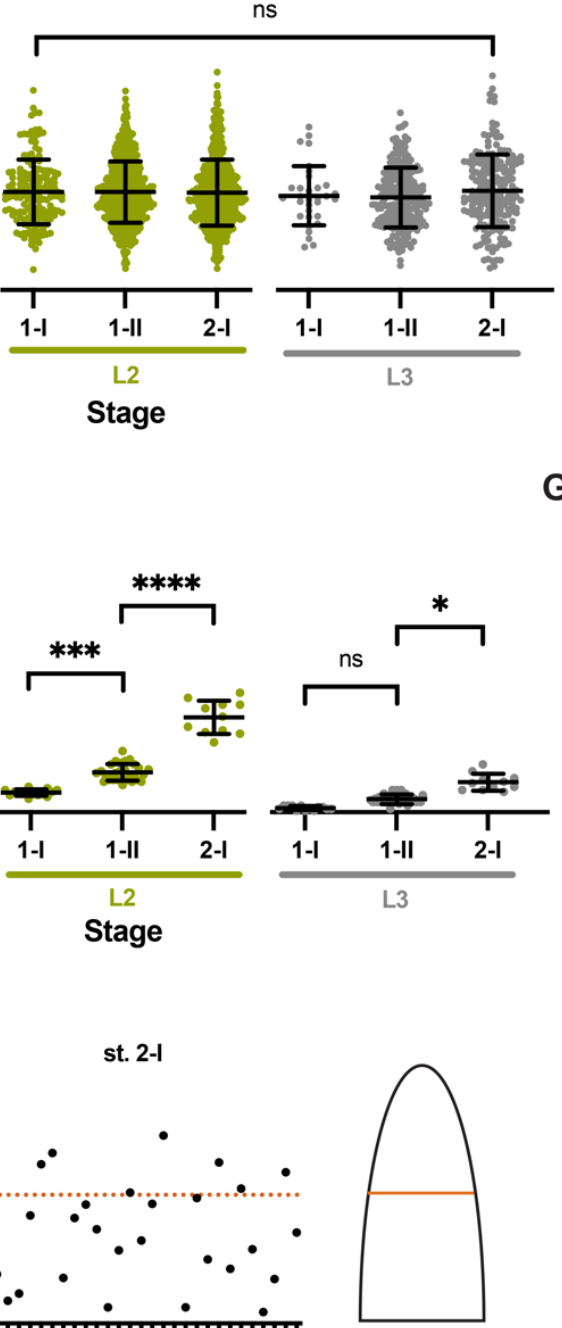

C

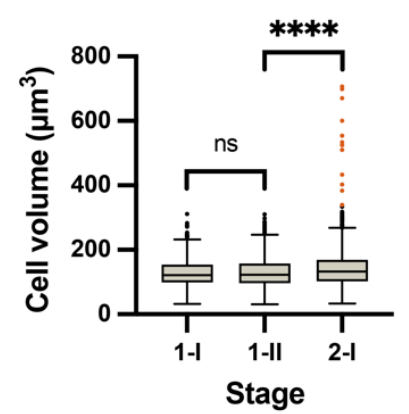

E

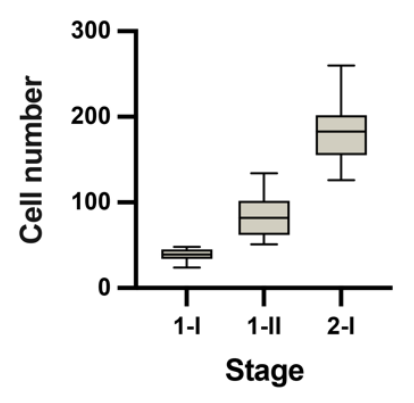

G
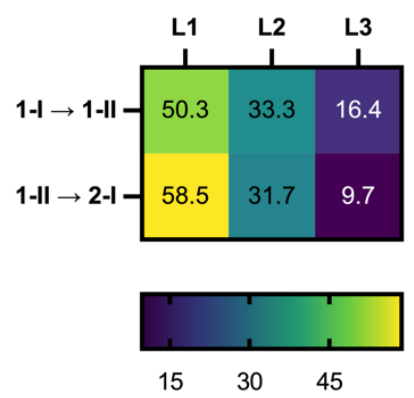

933 Fig 5. Cellular growth patterns in the ovule primordium. Different stages of wild-type

934 (Col-0) ovule primordia are analyzed. (A) 3D frontal plane view of ovule primordia

935 displaying the To-PRO-3 nuclear stain. Cells undergoing mitosis are outlined in red and marked by arrows. (B) Same section in (A) with an overlay of 3D labelled meshes. 
Arrows indicate mitotic figures (C) Comparison of cell volumes. Box and whiskers plots

938

939

940

941

942

943

944

945 are shown. Whiskers extend to $1.5 x$ the interquartile range from the 25 or 75 percentile, respectively (Tukey). (D) Comparison of cell volume between radial layers of different stages. (E) Comparison of cell numbers. Box and whiskers plots are shown. Whiskers extend to $1.5 \mathrm{x}$ the interquartile range from the 25 or 75 percentiles, respectively (Tukey).

(F) Comparison of cell numbers between radial layers of different stages. (G) Heatmap of percentage increase in number of cells per tissue compared to overall increase in number of cells. (H) Plot showing the relative distance along the proximal-distal axis of mitotic cells in a primordium. Relative distance was calculated by the following formula: reldist $=1-$ distance coordinate/mean length. The stages are indicated. Statistics in $(\mathrm{D}, \mathrm{F}):$ data are mean $\pm \mathrm{SD}$; significances: $* * * *, p<0.0001 ; * * *, p<0.0004 ; *, \mathrm{p}<0.02$. Ordinary one-way ANOVA followed by Tukey's multiple comparison test. Scale bars: $20 \mu \mathrm{m}$.

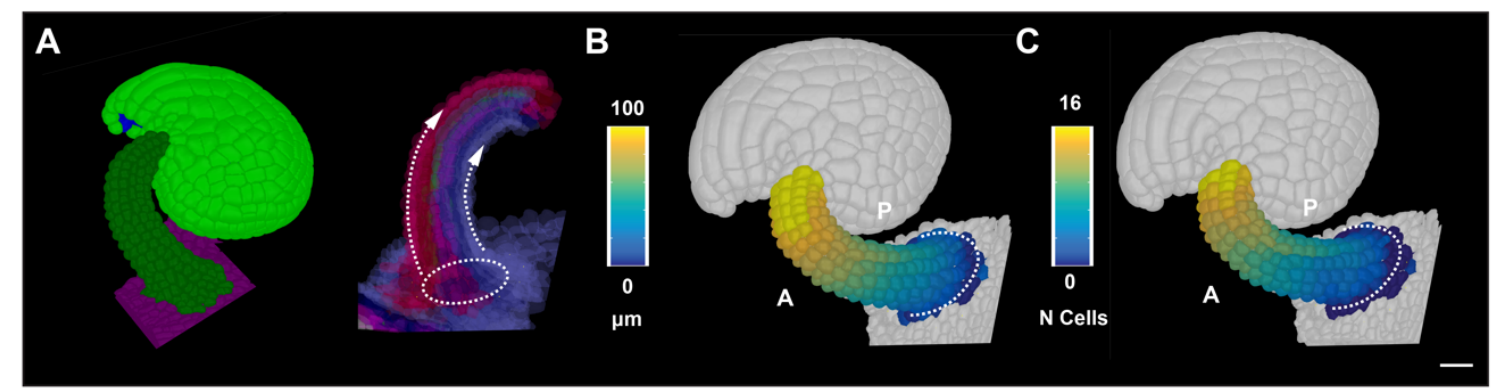

D

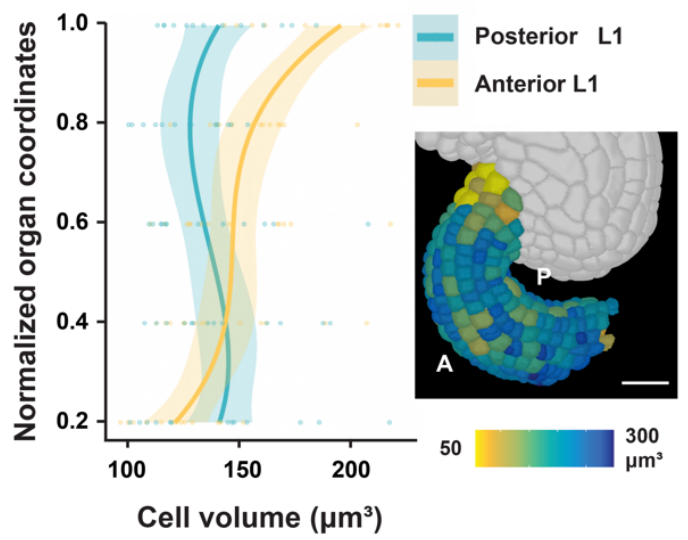

E

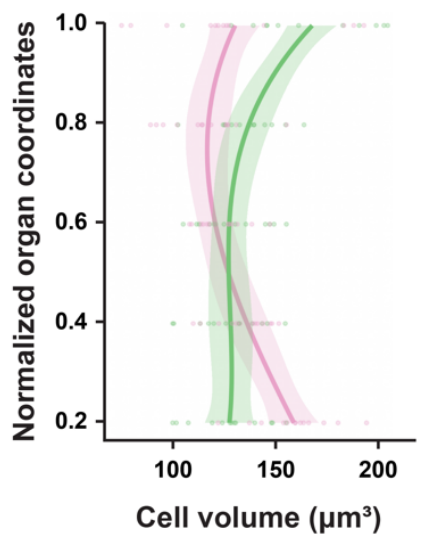

Cell volume $\left(\mu \mathrm{m}^{3}\right)$
Posterior L2

Anterior L2

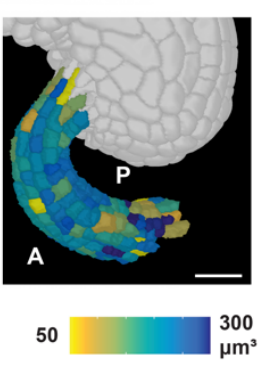


951 Fig 6. Cellular features of funiculus curvature. Wild-type (Col-0) ovules of stage 3-IV

952 are analyzed. (A) Left panel: Tilted side-view of a 3D cell mesh. Right panel: Semi-

953 transparent 3D mesh view of funiculus extracted from the 3D mesh of the organ. The

954 Bezier ring serving as origin is placed at the proximal base of the funiculus. The dashed

955 arrow lines indicate the coordinate direction along the anterior and posterior midlines.

$956(B, C)$ Same specimen as in (A). The anterior and posterior sides are marked. (B) Heatmap

957 of cell distances along the proximal-distal axis of the funiculus. (C) Heatmap of cell

958 numbers along the proximal-distal axis of the funiculus. White dotted line indicates the

959 coordinate origin as a ring. (D) Graph depicting cell volumes of anterior and posterior L1

960 cells in relation to the normalized proximal-distal position. The inset in the bottom right

961 corner shows a heat map of cell volume in the L1 of the funiculus. 14 3D digital ovules

962 were analyzed. Regression curves are cubic polynomials with 95\% confidence intervals

963 (shaded regions). Number of cells: $1568 \leq \mathrm{n} \leq 1768$. (E) Similar graph as in (D) revealing

964 cell volumes of anterior and posterior L2 cells. Number of cells: $1175 \leq n \leq 1352$. Scale

965 bars: $20 \mu \mathrm{m}$.

966 

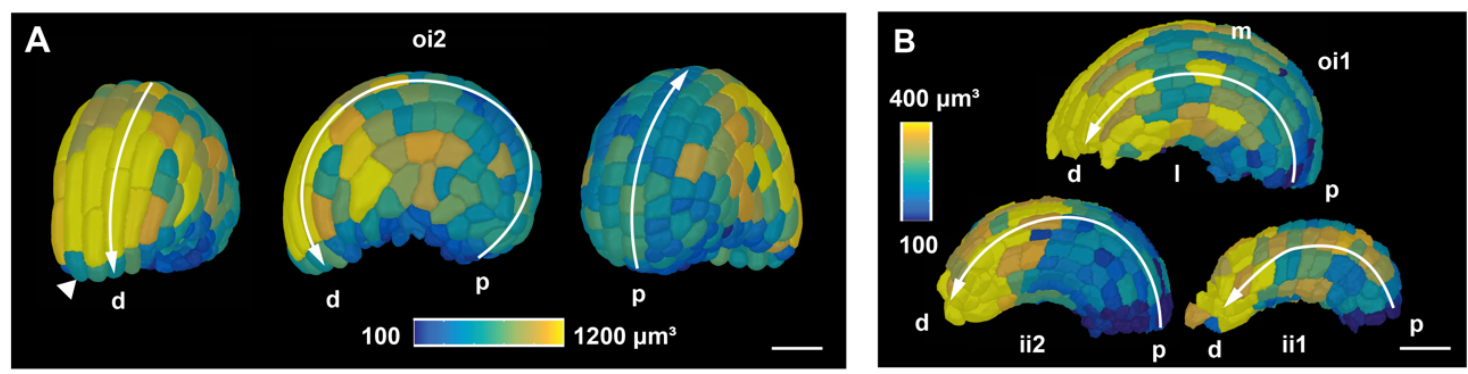

C

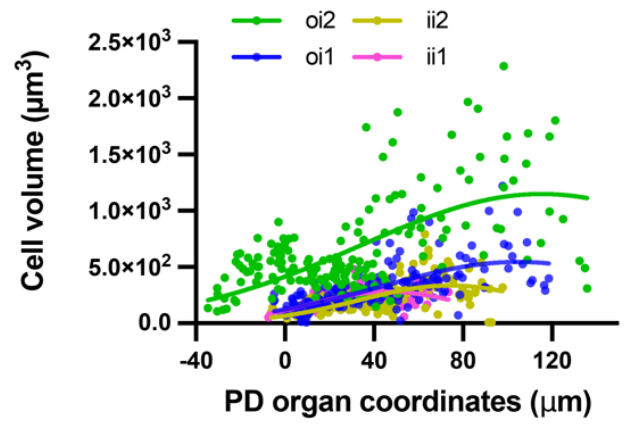

D

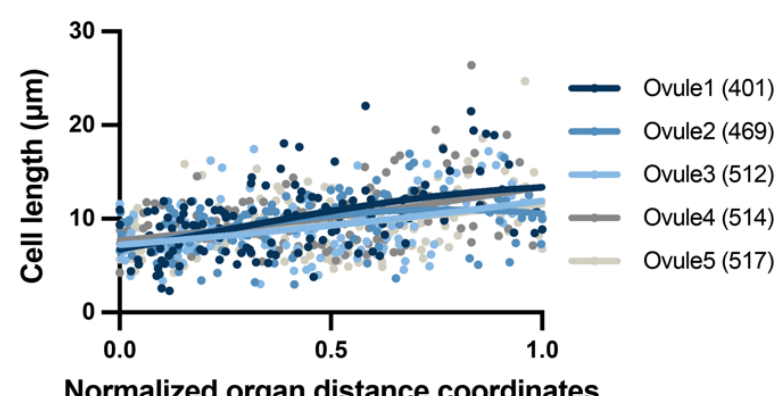

Fig 7.3D geometry of integument cells. Wild-type (Col-0) ovules of stage 3-IV are

analyzed. (A) Heatmap of oi2 cell volumes. Panels depict a tilted frontal view (left), side

view (center), and tilted back view (right). The proximal-distal axis is indicated. The

arrowhead marks a small tip cell. (B) Side views of the same specimen as in (A) showing

the 3D surface view of internal tissues. Heatmaps of the oi1, ii2, and ii1 layers, proximal-distal distance. Cells in the median oil layer of five ovules were analyzed. The respective nonlinear Gaussian regression curves are indicated. $126 \leq \mathrm{n} \leq 133$. Note the increase in cell length towards the distal end. Abbreviations: d, distal; ii1, inner layer of inner integument; ii2, outer layer of inner integument; 1, lateral; m, medial; oi1, inner

980 layer of outer integument; oi2, outer layer of outer integument; $p$, proximal.Scale bars:

$981 \quad(\mathrm{~A}, \mathrm{~B}) 20 \mu \mathrm{m}$. 


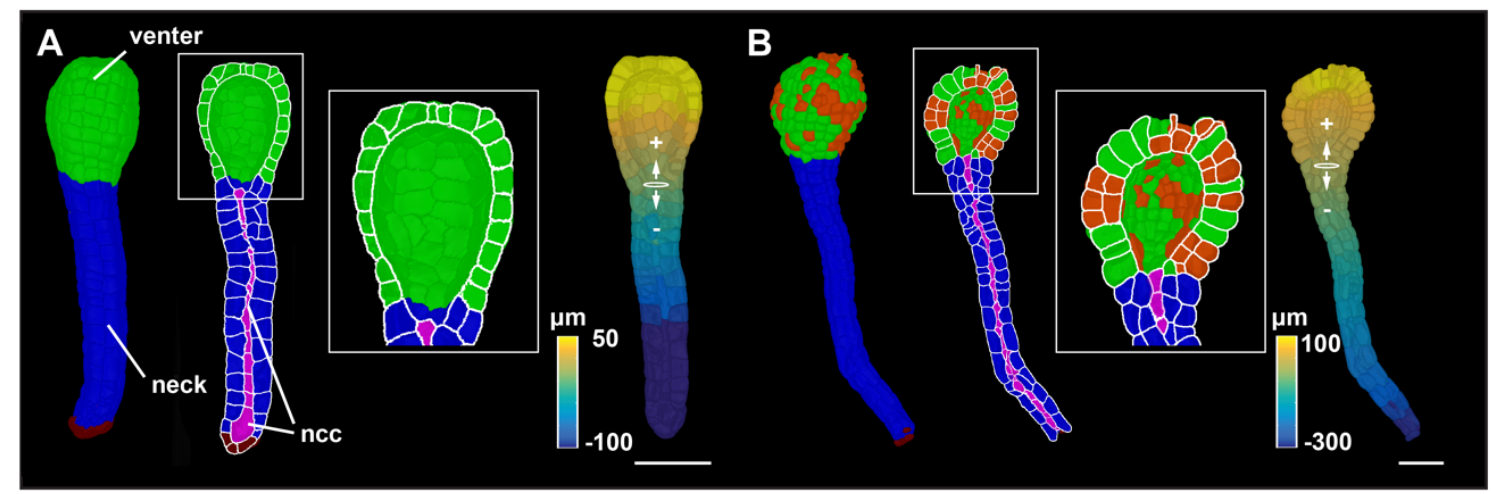

C

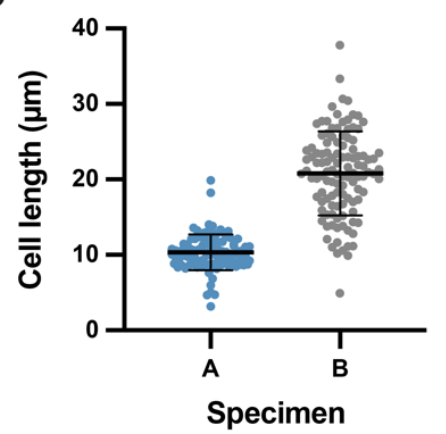

D

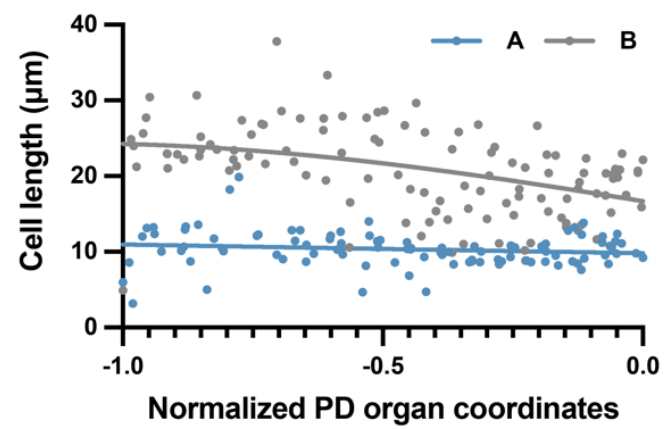

meshes of two different-stage archegonia are depicted. (A) Specimen A. The venter and neck are indicated. Left: 3D view. Center: An about mid-sagittal section. Right: Distance values along the central axis. The Bezier ring at the venter-neck boundary, which serves as the origin of the coordinate system, is indicated. (B) Specimen B. Identical arrangement as in (A). Highlighted white box represents the zoom view of the venter cells having undergone periclinal cell divisions marked in red. (C) Graph depicting the cell lengths of all neck cells of specimens A and B. The mean \pm SD is indicated. Specimen A: $\mathrm{n}=98$. Specimen B: $\mathrm{n}=114$. (D) Graph showing cell length of individual neck cells of the two specimens shown in $(\mathrm{C})$ in relation to their normalized position along the long axis of the neck. The tip of the neck is oriented to the left ( 0.0 corresponds to the ring position, the tip of the neck is at position -1.0, compare with Fig. 8B). Abbreviations: ncc, neck canal cells. Scale bars: (A,B) $50 \mu \mathrm{m}$. 

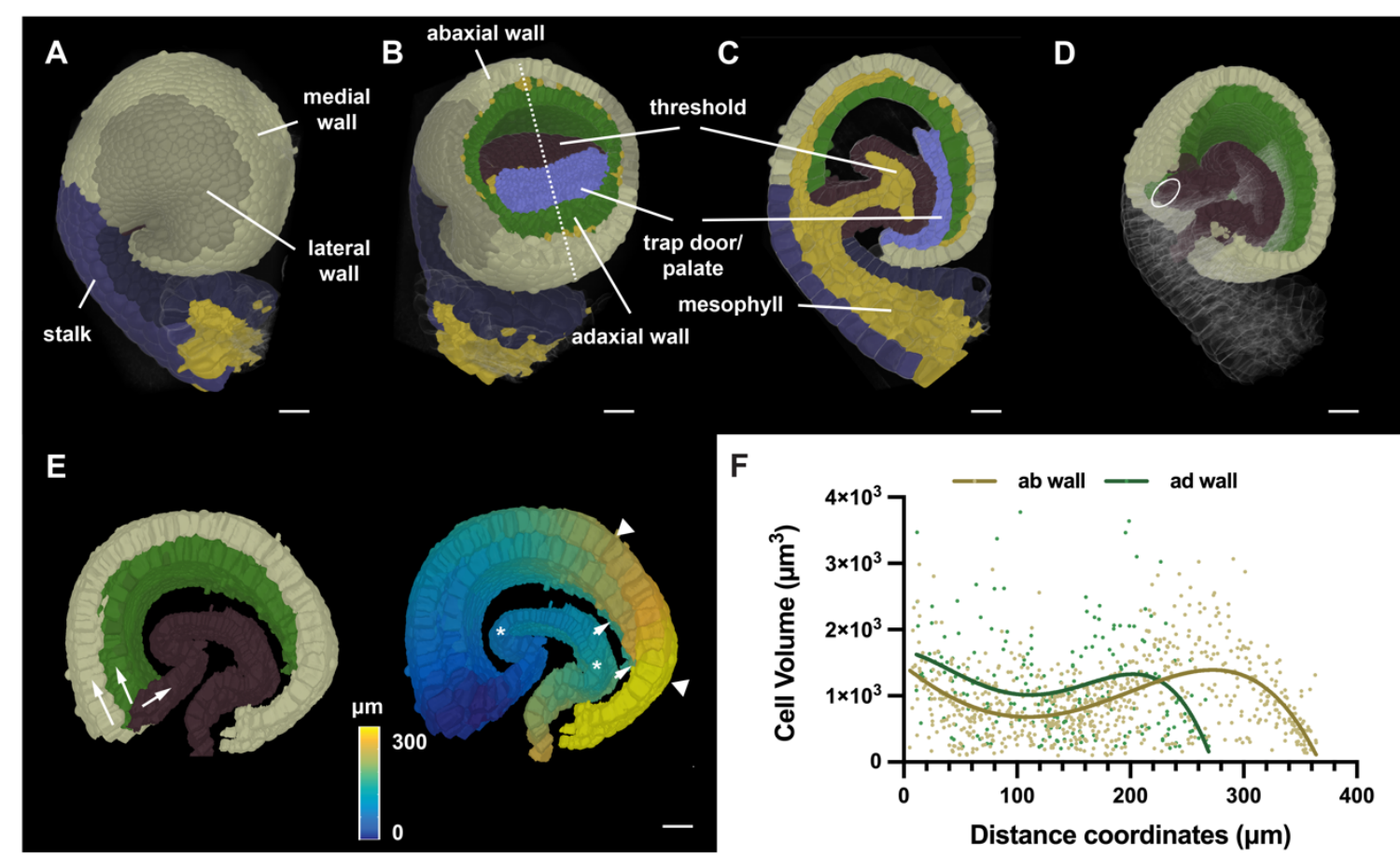

G

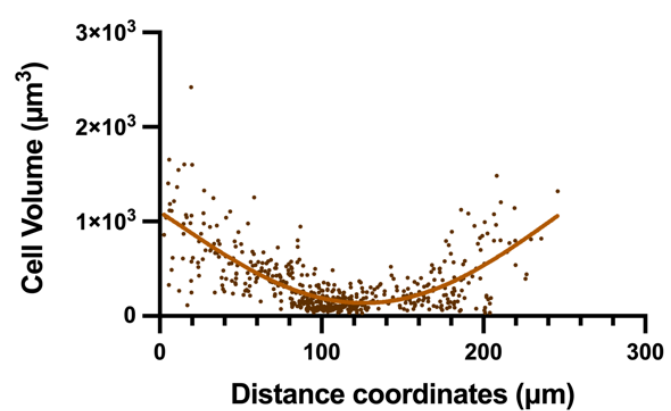

Fig 9. 3D digital Utricularia trap. A specimen 6 days after initiation is shown. (A) Side

view of the 3D cell mesh with annotation of various tissues. (B) Tilted view of (A) with part of the wall removed by a tangential clipping plane. The dashed line indicates the mid-sagittal section shown in (C). (C) Mid-sagittal section. (D) Slanted 3D view of (A) with half of the trap cut off at the mid-sagittal plane shown in (B). The trap door and palate domain were removed. The position of the Bezier ellipsoid is indicated. (E) Left panel: arrows indicate the direction of the distance coordinates through the epidermis of the abaxial and adaxial tissue of the wall and the threshold, respectively. Right panel:

1007 Heat map indicating distances. Wall and threshold are treated separately. Triangles mark the 240-320 $\mu \mathrm{m}$ interval of the abaxial wall. Arrows highlight the tapering end of the adaxial wall. Asterisks indicate the $80-150 \mu \mathrm{m}$ interval of the threshold. (F) Graph 
1010 displaying cell volume of epidermal trap cells in relation to their position. Values for the

1011 abaxial and adaxial wall are superimposed. The respective nonlinear regression curves

1012 fitting a fourth order polynomial function are indicated. ab wall: $\mathrm{n}=786$, ad wall: $\mathrm{n}=$

1013 231. (G). Graph displaying cell volume of threshold cells in relation to their position. The

1014 line marks a nonlinear regression curve. $\mathrm{n}=533$. Scale bars: $20 \mu \mathrm{m}$.

1015

1016 Tables

1017 Table 1. Layer-specific cellular growth characteristics in the ovule primordium

1018 anumber of 3D digital ovules scored: 14 (stage 1-I), 28 (stage 1-II), 11 (stage 2-I).

\begin{tabular}{|l|l|l|l|l|l|l|}
\hline Cell layer & \multicolumn{5}{|c|}{ Stage $^{\mathrm{a}}$} \\
\hline & \multicolumn{2}{|c|}{ I-I } & \multicolumn{2}{c|}{1 -II } & \multicolumn{2}{c|}{ 2-I } \\
\hline & Cell number & $\begin{array}{l}\text { Cell } \\
\text { volume }\end{array}$ & Cell number & $\begin{array}{l}\text { Cell } \\
\text { volume }\end{array}$ & Cell number & $\begin{array}{l}\text { Cell } \\
\text { volume }\end{array}$ \\
\hline L1 & $25.6 \pm 3.7$ & $115.6 \pm 35.7$ & $43.8 \pm 11.6$ & $115.2 \pm 36.8$ & $103.6 \pm 18.4$ & $129.9 \pm 44.5$ \\
\hline L2 & $11.6 \pm 1.9$ & $152.8 \pm 50.3$ & $23.4 \pm 4.9$ & $152.7 \pm 47.6$ & $55.6 \pm 9.7$ & $158.5 \pm 74.2$ \\
\hline L3 & $2.5 \pm 1.2$ & $146.8 \pm 45.9$ & $7.7 \pm 2.8$ & $144.1 \pm 46.6$ & $17.6 \pm 5.0$ & $154.7 \pm 56.5$ \\
\hline
\end{tabular}

$1019{ }^{b}$ Volumes are given in $\mu \mathrm{m}^{3}$.

1020 Values represent mean $\pm \mathrm{SD}$ 


\section{The annotation and analysis of complex 3D plant organs using 3DCordX}

Athul Vijayan ${ }^{1, \# a, \uparrow, ~ S o e r e n ~ S t r a u s s ~}{ }^{2, \uparrow}$, Rachele Tofanelli ${ }^{1}$, Tejasvinee Atul Mody ${ }^{1}$, Karen Lee ${ }^{3}$, Miltos Tsiantis ${ }^{2}$, Richard S. Smith ${ }^{2,3}$ and Kay Schneitz,*

${ }^{1}$ Plant Developmental Biology, TUM School of Life Sciences, Technical University of Munich, Freising, Germany

${ }^{2}$ Department of Comparative Developmental and Genetics, Max Planck Institute for Plant Breeding Research, Cologne, Germany

${ }^{3}$ The John Innes Centre, Norwich Research Park, Norwich, United Kingdom

${ }^{\text {\#a Current address }}$

Department of Comparative Developmental and Genetics, Max Planck Institute for Plant Breeding Research, Cologne, Germany

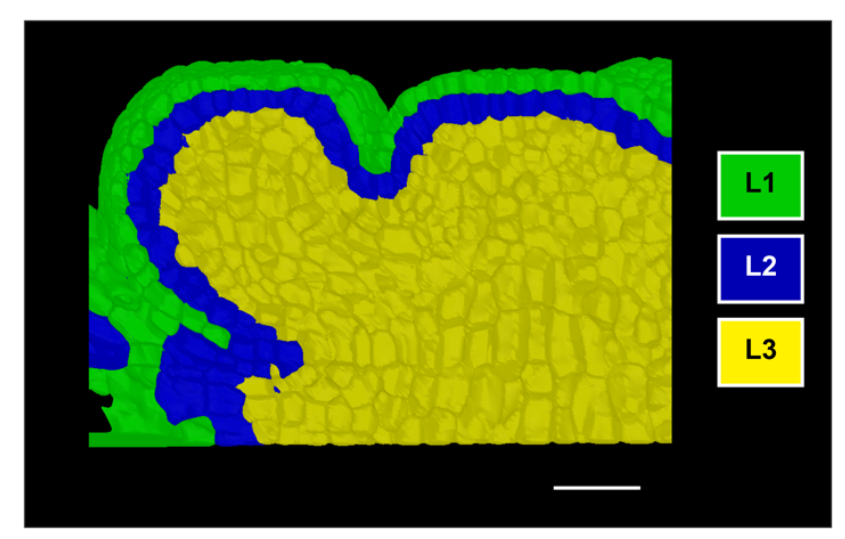

Fig S1. Automatic cell layer detection in the Arabidopsis shoot apical meristem.

The outside wall area ratio approach was applied to automatically identify the three tissue layers. Layers are labelled with respective colors. Scale bar $20 \mu \mathrm{m}$. 


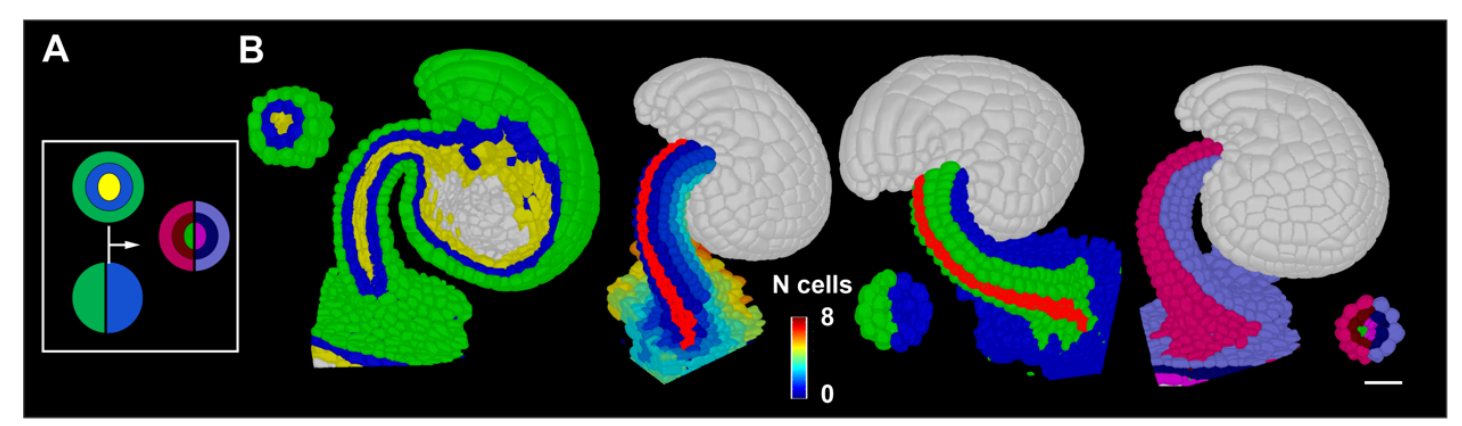

Fig. S2. Separation of funiculus into radial and anterior and posterior domains. A cartoon demonstrating the principle for the separation into radial L1, L2, L3 layers and anterior-posterior domains. (A) Results of L1, L2 and L3 tissue labels are combined with anterior and posterior labels to form the final tissue labels that separate individual radial layers and anterior-posterior tissues. (B) Figure demonstrating the step-by-step procedure for the detection of the anterior and posterior regions of the Arabidopsis funiculus. From left to right; first panel: L1, L2 and L3 labelled Arabidopsis ovule, a transverse section is shown at the top left; second panel: cells at roughly anterior midline are selected as origin (highlighted in red) and cell distance from these cells to other cells in funiculus are computed. Essentially the cell distance here represents the number of cells a cell is away from the anterior midline cells; third panel: the heatmap of cell distances from the second image is binned into two resulting in anterior and posterior tissue annotations; fourth panel: the results from first and third panel are merged to form the final tissue label that combines the information for L1, L2, L3 and anterior and posterior. This allows for example the distinction of anterior L1 and posterior L1 tissue. Scale bar $20 \mu \mathrm{m}$. 